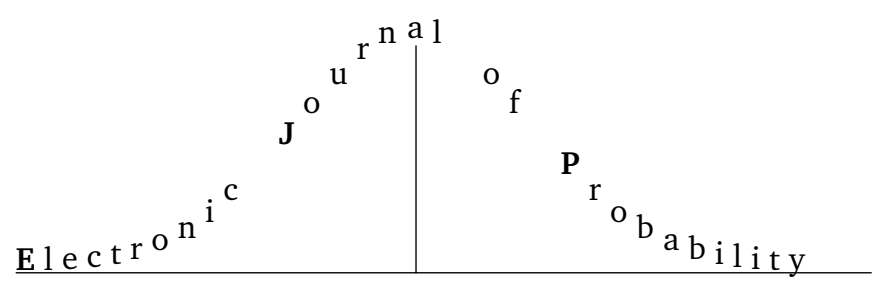

Vol. 14 (2009), Paper no. 61, pages 1790-1826.

Journal URL

http://www . math. washington.edu/ ejpecp/

\title{
Fractional Poisson Processes and Related Planar Random Motions
}

\author{
L.Beghin and E.Orsingher \\ Dipartimento di Statistica, Probabilità e Statistiche Applicate \\ "Sapienza" Università di Roma \\ p.le A.Moro 5 \\ 00185 Roma (Italy) \\ e-mail: enzo.orsingher@uniroma1.it, luisa.beghin@uniroma1.it
}

\begin{abstract}
We present three different fractional versions of the Poisson process and some related results concerning the distribution of order statistics and the compound Poisson process.

The main version is constructed by considering the difference-differential equation governing the distribution of the standard Poisson process, $N(t), t>0$, and by replacing the time-derivative with the fractional Dzerbayshan-Caputo derivative of order $v \in(0,1]$.

For this process, denoted by $\mathscr{N}_{v}(t), t>0$, we obtain an interesting probabilistic representation in terms of a composition of the standard Poisson process with a random time, of the form $\mathscr{N}_{v}(t)=N\left(\mathscr{T}_{2 v}(t)\right), t>0$. The time argument $\mathscr{T}_{2 v}(t), t>0$, is itself a random process whose distribution is related to the fractional diffusion equation.

We also construct a planar random motion described by a particle moving at finite velocity and changing direction at times spaced by the fractional Poisson process $\mathscr{N}_{v}$. For this model we obtain the distributions of the random vector representing the position at time $t$, under the condition of a fixed number of events and in the unconditional case.

For some specific values of $v \in(0,1]$ we show that the random position has a Brownian behavior (for $v=1 / 2$ ) or a cylindrical-wave structure (for $v=1$ ).
\end{abstract}

Key words: Fractional derivative; Fractional heat-wave equations; Finite velocity random motions; Cylindrical waves; Random velocity motions; Compound Poisson process; Order statistics; Mittag-Leffler function.. 
AMS 2000 Subject Classification: Primary 60G55; 26A33.

Submitted to EJP on December 2, 2008, final version accepted June 30, 2009. 


\section{Introduction}

Attempts to construct fractional versions of the Poisson process have been undertaken by Repin and Saichev (2000), Jumarie (2001) and Laskin (2003).

In analogy with the well-known fractional Brownian motion, the idea of passing from the usual Poisson process to a possible fractional version has inspired the papers by Wang et al. (2006)(2007). The process proposed by them is constructed as a stochastic integral with respect to the Poisson measure.

We follow here a completely different approach and we construct various types of fractional Poisson processes.

Our main results concern the fractional Poisson process $\mathscr{N}_{v}(t), t>0$, whose distribution $p_{k}=$ $\operatorname{Pr}\left\{\mathscr{N}_{v}(t)=k\right\}, k \geq 0$, solves the following equation

$$
\frac{d^{v} p_{k}}{d t^{v}}=-\lambda p_{k}+\lambda p_{k-1}, \quad k \geq 0,
$$

with $p_{-1}(t)=0$ and subject to the initial conditions

$$
p_{k}(0)= \begin{cases}1 & k=0 \\ 0 & k \geq 1\end{cases}
$$

We apply in (1.1) the definition of fractional derivative in the sense of Dzerbayshan-Caputo, that is, for $m \in \mathbb{N}$,

$$
\frac{d^{v}}{d t^{v}} u(t)=\left\{\begin{array}{ll}
\frac{1}{\Gamma(m-v)} \int_{0}^{t} \frac{1}{(t-s)^{1+v-m}} \frac{d^{m}}{d s^{m}} u(s) d s, & \text { for } m-1<v<m \\
\frac{d^{m}}{d t^{m}} u(t), \quad \text { for } v=m
\end{array},\right.
$$

(see Podlubny (1999), p.78).

We note that, for $v=1$, (1.1) coincides with the equation governing the homogeneous Poisson process, so that our results generalize the well-known distributions holding in the standard case.

The solution to (1.1)-(1.2) is expressed as the distribution of a process, which we will denote by $\mathscr{N}_{v}(t), t>0$. The presence of the fractional derivative in (1.1) implies a series of consequences, the most important being that $\mathscr{N}_{v}$ has dependent increments.

We will obtain various forms of the solution to (1.1): the first one is expressed as

$$
\begin{aligned}
\operatorname{Pr}\left\{\mathscr{N}_{v}(t)=k\right\} & =\sum_{r=k}^{\infty}(-1)^{r-k}\left(\begin{array}{l}
r \\
k
\end{array}\right) \frac{\left(\lambda t^{v}\right)^{r}}{\Gamma(v r+1)} \\
& =\frac{\left(\lambda t^{v}\right)^{k}}{k !} \sum_{r=0}^{\infty} \frac{(r+k) !}{r !} \frac{\left(-\lambda t^{v}\right)^{r}}{\Gamma(v(k+r)+1)}
\end{aligned}
$$

for $k \geq 0, t>0$.

The distribution (1.4) coincides with formula (25) of Laskin (2003), despite the latter being obtained as a solution to equation (1.1) with the fractional derivative defined in the Riemann-Liouville sense (instead of (1.3)). The additional impulse term appearing in equation (25) of Laskin (2003) justifies this coincidence. 
In Jumarie (2001) the fractional derivative is also meant in the Riemann-Liouville sense, but it is subject to a fractional initial condition, thus obtaining again formula (1.4). For a general reference on fractional calculus see Samko et al. (1993).

An alternative form of the distribution is given in terms of the generalized Mittag-Leffler function, which is defined (see Podlubny (1999), p.17) as

$$
E_{v, \mu}(x)=\sum_{k=0}^{\infty} \frac{x^{k}}{\Gamma(v k+\mu)}, \quad v, \mu>0, x \in \mathbb{R} .
$$

For small values of $k$ we have meaningful expression: for example, for $k=0$, we get that

$$
\operatorname{Pr}\left\{\mathscr{N}_{v}(t)=0\right\}=E_{v, 1}\left(-\lambda t^{v}\right),
$$

which represents the distribution of the waiting time of the first fractional Poisson event.

We are able to prove that the fractional Poisson process $\mathscr{N}_{v}(t), t>0$ with distribution (1.4) is related to the usual homogeneous Poisson process $N(t), t>0$ by means of the following relationship

$$
\operatorname{Pr}\left\{\mathscr{N}_{v}(t)=k\right\}=\int_{0}^{+\infty} \operatorname{Pr}\{N(s)=k\} \operatorname{Pr}\left\{\mathscr{T}_{2 v}(t) \in d s\right\}, \quad k \geq 0 .
$$

In (1.7) $\mathscr{T}_{2 v}(t), t>0$ is a random process with distribution $v_{2 v}=v_{2 v}(y, t)$ obtained by solving the following Cauchy problem (for a fractional diffusion equation)

$$
\left\{\begin{array}{l}
\frac{\partial^{2 v} v}{\partial t^{2 v}}=\frac{\partial^{2} v}{\partial y^{2}}, \quad t>0, y \in \mathbb{R} \\
v(y, 0)=\delta(y), \quad \text { for } 0<v<1
\end{array},\right.
$$

with the additional condition $v_{t}(y, 0)=0$, for $1 / 2<v<1$.

The relationship (1.7) means that the fractional Poisson process $\mathscr{N}_{v}$ has the following representation

$$
\mathscr{N}_{v}(t)=N\left(\mathscr{T}_{2 v}(t)\right)
$$

and thus it can be considered as a homogeneous Poisson process stopped at a random time $\mathscr{T}_{2 v}(t)$. On the basis of the previous results we construct, in Section 3, a planar random motion described by a particle moving at finite velocity $c$ and changing direction at times spaced by the fractional Poisson process $\mathscr{N}_{v}$. At each change of direction (at fractional Poisson instants) the new direction is chosen with uniform law in $[0,2 \pi]$. This has been our first motivation of the research of this paper.

In the standard case, when the process governing the changes of directions is the standard Poisson process, this model has been studied in Kolesnik and Orsingher (2005). The conditional distribution of the random vector $(X(t), Y(t)), t>0$ (representing the position of the moving particle at time $t$ ) is given by

$$
\begin{aligned}
& \operatorname{Pr}\{X(t) \in d x, Y(t) \in d y \mid N(t)=k\} \\
= & \left\{\begin{array}{l}
\delta\left(c^{2} t^{2}-\left(x^{2}+y^{2}\right)\right), \quad k=0 \\
\frac{k}{2 \pi(c t)^{k}}\left(c^{2} t^{2}-\left(x^{2}+y^{2}\right)\right)^{\frac{k}{2}-1} d x d y, \quad k \geq 1
\end{array}\right.
\end{aligned}
$$

for $t>0,(x, y) \in C_{c t}=\left\{x, y: x^{2}+y^{2} \leq c^{2} t^{2}\right\}$. 
We obtain here the analogue to (1.10) for the fractional case where $v \in(0,1]$. In particular, for the case $v=1 / 2$, the fractional process governing the changes of directions is $\mathscr{N}_{1 / 2}(t)=N(|B(t)|)$, since, in this case, equation (1.8) reduces to the heat-equation. We prove that the conditional distribution of the random vector $(X(|B(t)|), Y(|B(t)|)), t>0$ can be written as

$$
\begin{aligned}
& \operatorname{Pr}\left\{X(|B(t)|) \in d x, Y(|B(t)|) \in d y \mid \mathscr{N}_{\frac{1}{2}}(t)=k\right\} \\
= & \frac{d x d y}{B\left(\frac{1}{2}, \frac{k+1}{2}\right)} \int_{0}^{1} w^{-\frac{1}{2}}(1-w)^{\frac{k-1}{2}} \frac{e^{-\frac{x^{2}+y^{2}}{4 c^{2} t w}}}{4 \pi t w c^{2}} d w, \quad k \geq 0, \quad(x, y) \in \mathbb{R}^{2} .
\end{aligned}
$$

where $B(\alpha, \beta)$ denotes a Beta function of parameters $\alpha, \beta$ and $B(t), t>0$, is a standard Brownian motion, independent of $(X(t), Y(t))$. This means that the planar motion with a Brownian time can be regarded as a planar Brownian motion, whose volatility is itself random and possesses a Beta distribution depending on the number of changes of directions.

The unconditional version of the distribution of the planar motion takes interesting forms, especially in some particular cases. In general, for any $v \in(0,1]$, we have that

$$
\begin{aligned}
& \quad \operatorname{Pr}\left\{X\left(\mathscr{T}_{2 v}(t)\right) \in d x, Y\left(\mathscr{T}_{2 v}(t)\right) \in d y\right\} \\
& =\frac{d x d y}{\pi^{2}} \int_{0}^{1} \frac{u_{2 v}\left(x, y, t ; c^{2} w\right)}{w^{1 / 2}} d w \int_{0}^{1} \frac{d z}{\sqrt{z(1-z)}} \times \\
& \times\left[\frac{E_{v, 1}\left(-\lambda t^{v}\right)}{\sqrt{1-w}}+\frac{\pi \lambda t^{v}}{2 v} E_{v, v}\left(-\lambda t^{v}(1-\sqrt{(1-w)(1-z)})\right)\right],
\end{aligned}
$$

where $u_{2 v}=u_{2 v}\left(x, y, t ; \lambda^{2}\right)$ is the fundamental solution to the fractional planar wave equation (see (3.16) below).

In the special case of $v=1$, the previous expression reduces to the following very interesting form:

$$
\begin{aligned}
& \operatorname{Pr}\left\{X\left(\mathscr{T}_{2}(t)\right) \in d x, Y\left(\mathscr{T}_{2}(t)\right) \in d y\right\} \\
= & \frac{d x d y}{\pi c} \int_{0}^{t} \frac{\partial}{\partial z}\left[\frac{1_{C_{c z}}(x, y)}{\sqrt{c^{2} z^{2}-\left(x^{2}+y^{2}\right)}}\right] q(z ; t) d z, \quad|z|<t,
\end{aligned}
$$

where

$$
q(z ; t)=\frac{\lambda e^{-\lambda t}}{2} \sum_{k=0}^{\infty}\left(\frac{\lambda \sqrt{t^{2}-z^{2}}}{2}\right)^{k-1} \frac{d z}{\Gamma^{2}\left(\frac{k}{2}+\frac{1}{2}\right)}, \quad|z|<t .
$$

The distribution (1.14) is the marginal of the unconditional distribution of the planar random motion $(X(t), Y(t)), t>0$ (performed at velocity 1), given in formula (1.3) of Orsingher and De Gregorio (2007).

The kernel function in (1.13)

$$
\frac{d x d y}{2 \pi c} \frac{\partial}{\partial t}\left[\frac{1_{C_{c t}}(x, y)}{\sqrt{c^{2} t^{2}-\left(x^{2}+y^{2}\right)}}\right]=u_{1}\left(x, y, t ; c^{2}\right)
$$


emerges in the study of cylindrical waves, that is the analysis of the following problem

$$
\left\{\begin{array}{l}
\frac{\partial^{2} u}{\partial t^{2}}=c^{2}\left\{\frac{\partial^{2} u}{\partial x^{2}}+\frac{\partial^{2} u}{\partial y^{2}}\right\}, \quad t>0, x, y \in \mathbb{R} \\
u(x, y, 0)=\delta(x) \delta(y) \\
u_{t}(x, y, 0)=0
\end{array} .\right.
$$

Section 4 is devoted to the investigation of alternative forms of fractional Poisson processes. The first one, denoted by $\widehat{N}_{v}(t)$, is defined by generalizing to the case $v \in(0,1]$ the Poisson distribution as follows

$$
\operatorname{Pr}\left\{\widehat{N}_{v}(t)=k\right\}=\frac{(\lambda t)^{k}}{\Gamma(v k+1)} \frac{1}{E_{v, 1}(\lambda t)}, \quad k \geq 0
$$

so that the probability generating function reads

$$
G_{v}(u, t)=\mathbb{E} u^{\widehat{N}_{v}(t)}=\frac{E_{v, 1}(u \lambda t)}{E_{v, 1}(\lambda t)}, \quad 0 \leq u \leq 1 .
$$

As we will see below, for $v=1$, formulae (1.16) and (1.17) reduce to the corresponding results holding for the standard Poisson case. Also this version of fractional Poisson process does not possess the property of independence of increments.

We prove that the probability generating function (1.17) is a solution to the fractional equation

$$
\frac{\partial^{v} G_{v}\left(u^{v}, t\right)}{\partial u^{v}}=\lambda t G_{v}\left(u^{v}, t\right), \quad 0<v \leq 1 .
$$

Based on the model above, we construct a fractional counterpart of the Poisson compound process, defined (for any fixed $t$ ) as the sum of a random number $\widehat{N}_{v}(t)$ of i.i.d. r.v.'s and we derive its characteristic function.

Moreover, for the first two versions of fractional Poisson processes, we obtain some results concerning the maximum, minimum and order statistics of flows of i.i.d. r.v.'s paced by $\mathscr{N}_{v}$ and $\widehat{N}_{v}$.

In hydrology and seismology the sequence of catastrophic events can be successively represented by a fractional Poisson process (which is devoid of the lack of memory property of the usual Poisson process). The evaluation of the distribution of the maximum of i.i.d. r.v.'s, whose number is represented by a fractional Poisson process, is therefore of practical use in determining the probability of extremal events.

The third process, $\overline{\mathscr{N}}_{v}(t), t>0$, introduced here is constructed as a renewal process with interarrival times $U_{j}, j \geq 1$, possessing the distribution given in (1.6), that is

$$
\operatorname{Pr}\left\{U_{j}>t\right\}=\operatorname{Pr}\left\{T_{1}^{v}>t\right\}=\operatorname{Pr}\left\{\mathscr{N}_{v}(t)=0\right\}=E_{v, 1}\left(-\lambda t^{v}\right) .
$$

The distribution of $\bar{N}_{v}$ is expressed, for $m \geq 0$, as

$$
\operatorname{Pr}\left\{\overline{\mathscr{N}}_{v}(t)=m\right\}=\lambda \int_{0}^{t} d z \int_{0}^{\infty} f_{v}(z ; u)[\operatorname{Pr}\{N(u)=m-1\}-\operatorname{Pr}\{N(u)=m\}] d u,
$$

where $f_{v}(\cdot ; t)$ is the density of the stable r.v. $S_{v}(\mu, \beta, \sigma)$, where $\mu=0, \beta=1$ and $\sigma=$ $(t \cos \pi v / 2)^{1 / v}$. 


\section{A first form of the fractional Poisson process}

We construct the first type of fractional Poisson process (which we will denote by $\mathscr{N}_{v}(t), t>0$ ) by replacing, in the differential equations governing the distribution of the classical Poisson process, the standard derivatives by the fractional derivatives, defined in (1.3), i.e. in the Dzerbayshan-Caputo sense.

Therefore we are interested in the solution of

$$
\frac{d^{v} p_{k}(t)}{d t^{v}}=\lambda p_{k-1}(t)-\lambda p_{k}(t), \quad k \geq 0
$$

(with $p_{-1}(t)=0$ ) with initial conditions

$$
p_{k}(0)=\left\{\begin{array}{ll}
1 & k=0 \\
0 & k \geq 1
\end{array} .\right.
$$

The use of definition (1.3) permits us to avoid fractional derivatives in the initial conditions and to obtain different and more explicit expressions of the solution, from a probabilistic point of view.

The equation governing the probability generating function $G$ in the standard case, i.e.

$$
\frac{\partial G}{\partial t}=\lambda(u-1) G
$$

is then replaced by the following equation

$$
\frac{\partial^{v} G}{\partial t^{v}}=\lambda(u-1) G, \quad 0<v \leq 1
$$

subject to the initial condition $G(u, 0)=1$.

The Laplace transform of the solution to (2.4) becomes

$$
\begin{aligned}
\left(\mathscr{L} G_{v}\right)(u, \mu) & =\int_{0}^{+\infty} e^{-\mu t} G_{v}(u, t) d t \\
& =\frac{\mu^{v-1}}{\mu^{v}-\lambda(u-1)} .
\end{aligned}
$$

The inverse Laplace transform of (2.5) yields the following probability generating function

$$
G_{v}(u, t)=E_{v, 1}\left(\lambda(u-1) t^{v}\right) .
$$

The uniqueness of the solutions to (2.4) can be proved by taking two solutions $\left(G_{1}\right.$ and $\left.G_{2}\right)$ and by solving the Cauchy problem for $h(u, t)=G_{1}(u, t)-G_{2}(u, t)$, with initial condition $h(u, 0)=0$. The existence of the solution is a consequence of our analysis.

Much information can be extracted from (2.6): some corollaries are immediate, for example

$$
\begin{aligned}
\mathbb{E} \mathscr{N}_{v}(t) & =\frac{\lambda t^{v}}{\Gamma(v+1)} \\
\operatorname{Var} \mathscr{N}_{v}(t) & =\frac{\lambda t^{v}}{\Gamma(v+1)}+\frac{\left(\lambda t^{v}\right)^{2}}{v}\left\{\frac{1}{\Gamma(2 v)}-\frac{1}{v \Gamma^{2}(v)}\right\}
\end{aligned}
$$




$$
\mathbb{E}\left\{\mathscr{N}_{v}(t)\left[\mathscr{N}_{v}(t)-1\right] \ldots\left[\mathscr{N}_{v}(t)-r+1\right]\right\}=\frac{\left(\lambda t^{v}\right)^{r} r !}{\Gamma(v r+1)} \quad r \geq 1
$$

We note that the usual equality between the mean value and the variance does not hold for this model, while, for $v=1$, it can be checked that $\mathbb{E} \mathscr{N}_{1}(t)=\operatorname{Var} \mathscr{N}_{1}(t)$.

The most interesting result is the distribution function related to (2.6), which can be presented as follows

$$
\begin{aligned}
\operatorname{Pr}\left\{\mathscr{N}_{v}(t)=k\right\} & =\frac{\left(\lambda t^{v}\right)^{k}}{k !} \sum_{r=0}^{\infty} \frac{(r+k) !}{r !} \frac{\left(-\lambda t^{v}\right)^{r}}{\Gamma(v(k+r)+1)} \\
& =\sum_{r=k}^{\infty}(-1)^{r-k}\left(\begin{array}{l}
r \\
k
\end{array}\right) \frac{\left(\lambda t^{v}\right)^{r}}{\Gamma(v r+1)} \\
& =\frac{1}{k !} \sum_{r=k}^{\infty} \frac{(-1)^{r-k}}{(r-k) !} \mathbb{E}\left\{\mathscr{N}_{v}(t) \ldots\left[\mathscr{N}_{v}(t)-r+1\right]\right\} .
\end{aligned}
$$

For small values of $k$ it is possible to write the distribution (2.10) in terms of Mittag-Leffler functions as follows

$$
\begin{aligned}
\operatorname{Pr}\left\{\mathscr{N}_{v}(t)=0\right\}= & E_{v, 1}\left(-\lambda t^{v}\right) \\
\operatorname{Pr}\left\{\mathscr{N}_{v}(t)=1\right\}= & \frac{\lambda t^{v}}{v} E_{v, v}\left(-\lambda t^{v}\right) \\
\operatorname{Pr}\left\{\mathscr{N}_{v}(t)=2\right\}= & \frac{\left(\lambda t^{v}\right)^{2}}{2 ! v^{2}}\left\{(1-v) E_{v, 2 v}\left(-\lambda t^{v}\right)+E_{v, 2 v-1}\left(-\lambda t^{v}\right)\right\} \\
\operatorname{Pr}\left\{\mathscr{N}_{v}(t)=3\right\}= & \frac{\left(\lambda t^{v}\right)^{3}}{3 ! v^{3}}\left\{2(1-v)\left(\frac{1}{2}-v\right) E_{v, 3 v}\left(-\lambda t^{v}\right)+\right. \\
& \left.+3(1-v) E_{v, 3 v-1}\left(-\lambda t^{v}\right)+E_{v, 3 v-2}\left(-\lambda t^{v}\right)\right\} .
\end{aligned}
$$

We derive now an explicit representation of the solution to (2.1)-(2.2), which suggests an interesting probabilistic interpretation of the related process. The next result represents also an important link between the fractional Poisson process and the fractional diffusion equations.

Let us denote by $v_{2 v}=v_{2 v}(y, t)$ the solution to the following Cauchy problem

$$
\left\{\begin{array}{l}
\frac{\partial^{2 v} v}{\partial t^{2 v}}=\frac{\partial^{2} v}{\partial y^{2}}, \quad t>0, y \in \mathbb{R} \\
v(y, 0)=\delta(y), \quad \text { for } 0<v<1
\end{array},\right.
$$

with the additional condition

$$
v_{t}(y, 0)=0, \quad \text { for } 1 / 2<v<1 .
$$

The condition (2.13) is due to the application of the Laplace transform of the fractional derivative (see Podlubny (1999), formula (2.253), p.106).

It is well-known that

$$
v_{2 v}(y, t)=\frac{1}{2 t^{v}} W_{-v, 1-v}\left(-|y| t^{-v}\right), \quad t>0, y \in \mathbb{R},
$$


where

$$
W_{\alpha, \beta}(x)=\sum_{k=0}^{\infty} \frac{x^{k}}{k ! \Gamma(\alpha k+\beta)}, \quad \alpha>-1, \beta>0, x \in \mathbb{R} .
$$

Theorem 2.1 Let

$$
\bar{v}_{2 v}(y, t)=\left\{\begin{array}{l}
2 v_{2 v}(y, t), \quad y>0 \\
0, \quad y<0
\end{array}\right.
$$

be the folded solution to (2.12), then the probability generating function of the fractional Poisson process $\mathscr{N}_{v}(t), t>0$ can be written as

$$
G_{v}(u, t)=\int_{0}^{+\infty} e^{\lambda y(u-1)} \bar{v}_{2 v}(y, t) d y .
$$

The corresponding distribution reads, for $k \geq 0$,

$$
\begin{aligned}
\operatorname{Pr}\left\{\mathscr{N}_{v}(t)=k\right\} & =\int_{0}^{+\infty} e^{-\lambda y} \frac{(\lambda y)^{k}}{k !} \bar{v}_{2 v}(y, t) d y \\
& =\operatorname{Pr}\left\{N\left(\mathscr{T}_{2 v}(t)\right)=k\right\},
\end{aligned}
$$

where $\mathscr{T}_{2 v}(t), t>0$ represents a random time with transition density given in (2.15).

Proof The Laplace transform (2.5) can be written as

$$
\left(\mathscr{L} G_{v}\right)(u, \mu)=\mu^{v-1} \int_{0}^{+\infty} e^{-y \mu^{v}} e^{\lambda y(u-1)} d y .
$$

We note that

$$
\mu^{v-1}=\int_{0}^{+\infty} e^{-\mu t} \frac{t^{-v}}{\Gamma(1-v)} d t
$$

while

$$
e^{-y \mu^{v}}=\int_{0}^{+\infty} e^{-\mu z} f_{v}(z ; y) d z, \quad 0<v<1, y>0
$$

where $f_{v}(\cdot ; y)$ is a stable law $S_{v}(\mu, \beta, \sigma)$ of order $v$, with parameters $\mu=0, \beta=1$ and $\sigma=$ $\left(y \cos \frac{\pi v}{2}\right)^{\frac{1}{v}}$.

Therefore, by using together (2.18) and (2.19), we obtain the inverse Laplace transform as

$$
G_{v}(u, t)=\frac{1}{\Gamma(1-v)} \int_{0}^{+\infty} d y \int_{0}^{t}(t-w)^{-v} f_{v}(w ; y) e^{\lambda y(u-1)} d w
$$

It has been proved in Orsingher and Beghin (2004) that the solution $v_{2 v}$ to (2.12) can be expressed as

$$
v_{2 v}(y, t)=\frac{1}{2 \Gamma(1-v)} \int_{0}^{t}(t-w)^{-v} f_{v}(w ;|y|) d w, \quad y \in \mathbb{R}
$$

so that we arrive at (2.16), by folding (2.21). Formula (2.17) now easily follows. 
Remark 2.1 It is easy to check that (2.17) is non-negative and sums up to zero.

We note that, in particular for $v=1 / 2$, the random time $\mathscr{T}_{1}(t), t>0$ becomes a reflecting Brownian motion, since, in this case, equation (2.12) reduces to the heat-equation

$$
\left\{\begin{array}{l}
\frac{\partial v}{\partial t}=\frac{\partial^{2} v}{\partial y^{2}}, \quad t>0, y \in \mathbb{R} \\
v(y, 0)=\delta(y)
\end{array}\right.
$$

and the solution $v_{1}(y, t)$ is the density of a Brownian motion $B(t), t>0$ with infinitesimal variance 2. After folding up the solution, we find the following density

$$
\begin{aligned}
\operatorname{Pr}\left\{\mathscr{N}_{1 / 2}(t)=k\right\} & =\int_{0}^{+\infty} e^{-\lambda y} \frac{(\lambda y)^{k}}{k !} \frac{e^{-y^{2} / 4 t}}{\sqrt{\pi t}} d y \\
& =\operatorname{Pr}\{N(|B(t)|)=k\},
\end{aligned}
$$

so that $\mathscr{N}_{1 / 2}$ coincides with a standard Poisson process at a Brownian time.

Remark 2.2 We can check that, by replacing (2.14) into (2.16) we retrieve (2.6). We apply the following representations in terms of integrals on the Hankel contour $\mathrm{Ha}$ of the Wright and MittagLeffler functions, respectively:

$$
\begin{aligned}
W_{-v, 1-v}(x) & =\frac{1}{2 \pi i} \int_{H a} \frac{e^{z+x z^{v}}}{z^{1-v}} d z \\
E_{v, 1}(x) & =\frac{1}{2 \pi i} \int_{H a} \frac{e^{z} z^{v-1}}{z^{v}-x} d z .
\end{aligned}
$$

For a plot of the Hankel contour see Fig.1.

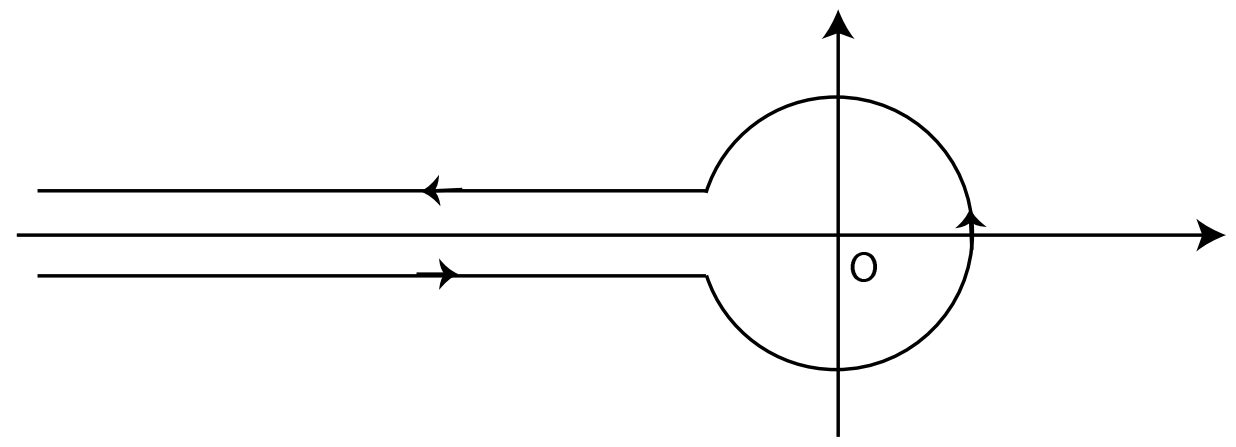

Figure 1: The Hankel contour

Therefore we achieve 


$$
\begin{aligned}
G_{v}(u, t) & =\int_{0}^{+\infty} e^{\lambda y(u-1)} \frac{1}{t^{v}} W_{-v, 1-v}\left(-y t^{-v}\right) d y \\
& =\int_{0}^{+\infty} e^{\lambda y(u-1)} \frac{d y}{t^{v}} \frac{1}{2 \pi i} \int_{H a} \frac{e^{z-y z^{v} t^{-v}}}{z^{1-v}} d z \\
& =\frac{t^{-v}}{2 \pi i} \int_{H a} \frac{e^{z}}{z^{1-v}} d z \int_{0}^{+\infty} e^{\lambda y(u-1)-y z^{v} t^{-v}} d y \\
& =\frac{t^{-v}}{2 \pi i} \int_{H a} \frac{e^{z} z^{v-1}}{z^{v} t^{-v}-\lambda(u-1)} d z \\
& =E_{v, 1}\left(\lambda(u-1) t^{v}\right)
\end{aligned}
$$

Remark 2.3 We derive from (2.17) an alternative expression of the distribution of the fractional Poisson process (in view of (2.14)), as follows:

$$
\begin{aligned}
& \operatorname{Pr}\left\{\mathscr{N}_{v}(t)=k\right\} \\
= & \int_{0}^{+\infty} e^{-\lambda y} \frac{(\lambda y)^{k}}{k !} t^{-v} \sum_{m=0}^{\infty} \frac{\left(-y t^{-v}\right)^{m}}{m ! \Gamma(-v m+1-v)} d y \\
= & \sum_{m=0}^{\infty} \frac{\lambda^{k} t^{-v-v m}(-1)^{m}}{k ! m ! \Gamma(-v m+1-v)} \int_{0}^{+\infty} e^{-\lambda y} y^{k+m} d y \\
= & \sum_{m=0}^{\infty} \frac{\lambda^{k} t^{-v(1+m)}(-1)^{m} \Gamma(k+m+1)}{\lambda^{k+m+1} k ! m ! \Gamma(-v m+1-v)} \\
= & \frac{t^{-v}}{\lambda} \sum_{m=0}^{\infty}\left(\begin{array}{c}
k+m \\
k
\end{array}\right) \frac{\left(-t^{v} \lambda\right)^{-m}}{\Gamma(-v m+1-v)},
\end{aligned}
$$

where the dependence on $k$ is limited to the binomial coefficient. It is easy to check in this form that the distribution (2.25) sums up to one with respect to $k$, as follows:

$$
\begin{aligned}
& \sum_{k=0}^{\infty} \operatorname{Pr}\left\{\mathscr{N}_{v}(t)=k\right\}=[k+m=r] \\
= & \frac{t^{-v}}{\lambda} \sum_{m=0}^{\infty} \sum_{r=m}^{\infty}\left(\begin{array}{l}
r \\
m
\end{array}\right) \frac{\left(-t^{v} \lambda\right)^{-m}}{\Gamma(-v m+1-v)}=\frac{t^{-v}}{\lambda} \sum_{r=0}^{\infty} \sum_{m=0}^{r}\left(\begin{array}{c}
r \\
m
\end{array}\right)\left(-t^{v} \lambda\right)^{-m} \frac{1}{2 \pi i} \int_{H a} e^{z} z^{v-1+v m} d z \\
= & \frac{t^{-v}}{\lambda} \sum_{r=0}^{\infty} \frac{1}{2 \pi i} \int_{H a} e^{z} z^{v-1} d z \sum_{m=0}^{r}\left(\begin{array}{c}
r \\
m
\end{array}\right)\left(-\lambda^{-1} t^{-v} z^{v}\right)^{m} \\
= & \frac{t^{-v}}{\lambda} \frac{1}{2 \pi i} \int_{H a} e^{z} z^{v-1} d z \sum_{r=0}^{\infty}\left(1-\frac{z^{v}}{\lambda t^{v}}\right)^{r}=\frac{t^{-v}}{\lambda} \frac{1}{2 \pi i} \int_{H a} e^{z} z^{v-1} \frac{d z}{1-\left(1-\frac{z^{v}}{\lambda t^{v}}\right)} \\
= & \frac{1}{2 \pi i} \int_{H a} e^{z} z^{-1} d z=\frac{1}{\Gamma(1)}=1,
\end{aligned}
$$


where, in the last step, we applied the contour-integral representation of the reciprocal of the Gamma function, i.e.

$$
\frac{1}{\Gamma(v)}=\frac{1}{2 \pi i} \int_{H a} e^{u} u^{-v} d u .
$$

The expression (2.25) suggests another useful representation of the solution in terms of derivatives of Mittag-Leffler functions, which generalizes the particular cases obtained in (2.11):

$$
\begin{aligned}
& \operatorname{Pr}\left\{\mathscr{N}_{v}(t)=k\right\} \\
= & \frac{t^{-v}}{\lambda} \sum_{m=0}^{\infty} \frac{(k+m)(k+m-1) \ldots(m+1)}{k !} \frac{\left(-t^{v} \lambda\right)^{-m}}{\Gamma(-v m+1-v)} \\
= & \frac{t^{-v}}{\lambda k !} \frac{d^{k}}{d s^{k}}\left[\sum_{m=0}^{\infty} s^{m+k} \frac{\left(-t^{v} \lambda\right)^{-m}}{\Gamma(-v m+1-v)}\right]_{s=1} \\
= & \frac{t^{-v}}{\lambda k !} \frac{d^{k}}{d s^{k}}\left[s^{k} E_{-v, 1-v}\left(-\frac{s}{\lambda t^{v}}\right)\right]_{s=1} \\
= & \frac{1}{k !} \frac{d^{k}}{d s^{k}}\left[s^{k-1} E_{v, 1}\left(-\frac{\lambda t^{v}}{s}\right)\right]_{s=1},
\end{aligned}
$$

where, in the last step we have applied (5.1) in Appendix.

For $k=0,1,2,3$ we obtain again formulae (2.11): for example, for $k=2$ and, by considering result (5.2) in Appendix, we have that

$$
\begin{aligned}
& \operatorname{Pr}\left\{\mathscr{N}_{v}(t)=2\right\} \\
= & \frac{1}{2} \frac{d^{2}}{d s^{2}}\left[s E_{v, 1}\left(-\frac{\lambda t^{v}}{s}\right)\right]_{s=1} \\
= & \frac{1}{2} \frac{d}{d s}\left[E_{v, 1}\left(-\frac{\lambda t^{v}}{s}\right)+\frac{1}{v} E_{v, v}\left(-\frac{\lambda t^{v}}{s}\right) \frac{\lambda t^{v}}{s}\right]_{s=1} \\
= & \frac{1}{2}\left[\frac{\lambda t^{v}}{v s} E_{v, v}\left(-\frac{\lambda t^{v}}{s}\right)-\frac{\lambda t^{v}}{v s^{2}} E_{v, v}\left(-\frac{\lambda t^{v}}{s}\right)\right]_{s=1}+ \\
& +\frac{1}{2}\left[-\frac{\lambda t^{v}}{v s^{3}}\left[\frac{d}{d x} E_{v, v}(x)\right]_{x=-\frac{\lambda t^{v}}{s}}\right]_{s=1} \\
= & \frac{\lambda^{2} t^{2 v}}{2 v^{2}}\left[E_{v, 2 v-1}\left(-\lambda t^{v}\right)+(1-v) E_{v, 2 v}\left(-\lambda t^{v}\right)\right] .
\end{aligned}
$$

The case $k=3$ can be derived by performing similar steps and applying (5.1) and (5.3). 
Remark 2.4 We can evaluate the Laplace transform of (2.17) as follows

$$
\begin{aligned}
& \int_{0}^{+\infty} e^{-\mu t} d t \int_{0}^{+\infty} e^{-\lambda y} \frac{(\lambda y)^{k}}{k !} t^{-v} \sum_{m=0}^{\infty} \frac{\left(-y t^{-v}\right)^{m}}{m ! \Gamma(-v m+1-v)} d y \\
= & \int_{0}^{+\infty} e^{-\lambda y} \frac{(\lambda y)^{k}}{k !} \sum_{m=0}^{\infty} \frac{(-y)^{m}}{m !} \mu^{v m+v-1} d y \\
= & \mu^{v-1} \frac{\lambda^{k}}{k !} \int_{0}^{+\infty} e^{-\lambda y-y \mu^{v}} y^{k} d y \\
= & \mu^{v-1} \frac{\lambda^{k}}{k !} \frac{\Gamma(k+1)}{\left(\lambda+\mu^{v}\right)^{k+1}}=\mu^{v-1} \frac{\lambda^{k}}{\left(\lambda+\mu^{v}\right)^{k+1}} .
\end{aligned}
$$

A further check stems from the fact that

$$
\begin{aligned}
& \int_{0}^{+\infty} e^{-\mu t} G_{v}(u, t) d t \\
= & \int_{0}^{+\infty} e^{-\mu t} \sum_{k=0}^{\infty} u^{k} \operatorname{Pr}\left\{\mathscr{N}_{v}(t)=k\right\} d t \\
= & \mu^{v-1} \sum_{k=0}^{\infty} u^{k} \frac{\lambda^{k}}{\left(\lambda+\mu^{v}\right)^{k+1}} \\
= & \frac{\mu^{v-1}}{\lambda+\mu^{v}} \frac{1}{1-\frac{u \lambda}{\lambda+\mu^{v}}},
\end{aligned}
$$

which coincides with $(2.5)$.

Remark 2.5 We note that the fractional Poisson process $\mathscr{N}_{v}$ loses the lack of memory property of the homogeneous Poisson process: indeed it is easy to check that it possesses dependent increments, since, from (2.6), we get

$$
\begin{aligned}
G_{v}\left(u, t_{1}+t_{2}\right) & =\mathbb{E} u^{\mathscr{N}_{v}\left(t_{1}+t_{2}\right)} \neq \mathbb{E} u^{\mathscr{N}_{v}\left(t_{1}\right)} \mathbb{E} u^{\mathscr{N}_{v}\left(t_{2}\right)} \\
& =G_{v}\left(u, t_{1}\right) G_{v}\left(u, t_{2}\right) .
\end{aligned}
$$

We consider now a sequence of a random number of i.i.d. non-negative random variables $X_{i}, i \geq$ 1 with distribution function $F(w)=\operatorname{Pr}\left(X_{i}<w\right), i \geq 1$, under the assumption that this random number is represented by $\mathscr{N}_{v}(t)$. We will obtain the distribution of the $k$-th order statistic $X_{(k)}^{\mathscr{N}_{v}(t)}, k \geq$ 1 , conditioned on the event $\mathscr{N}_{v}(t) \geq k$.

Theorem 2.2 Let $\mathscr{N}_{v}^{\lambda}(t), t>0$ be a fractional Poisson process of parameter $\lambda$, then the conditional 
distribution of the $k$-th order statistic $X_{(k)}^{\mathscr{N}_{v}(t)}, k \geq 1,0<v<1$, reads

$$
\begin{aligned}
& \operatorname{Pr}\left\{X_{(k)}^{\mathscr{N}_{v}(t)}<z \mid \mathscr{N}_{v}(t) \geq k\right\} \\
= & \frac{\sum_{l=k}^{\infty} \frac{\left(-\lambda t^{v} F(z)\right)^{l}}{\Gamma(v l+1)} \sum_{j=k}^{l}\left(\begin{array}{l}
l \\
j
\end{array}\right)(-1)^{j}}{\sum_{l=k}^{\infty} \frac{\left(-\lambda t^{v}\right)^{l}}{\Gamma(v l+1)} \sum_{j=k}^{l}\left(\begin{array}{l}
l \\
j
\end{array}\right)(-1)^{j}}=\frac{\operatorname{Pr}\left\{\mathscr{N}_{v}^{\lambda F(z)}(t) \geq k\right\}}{\operatorname{Pr}\left\{\mathscr{N}_{v}^{\lambda}(t) \geq k\right\}} .
\end{aligned}
$$

Proof We recall that in the standard case the $k$-th order statistics possess the following distribution, for $1 \leq k \leq n$,

$$
\operatorname{Pr}\left\{X_{(k)}^{n}<z\right\}=\sum_{j=k}^{n}\left(\begin{array}{l}
n \\
j
\end{array}\right) F^{j}(z)[1-F(z)]^{n-j},
$$

therefore in this case we have that

$$
\begin{aligned}
& \operatorname{Pr}\left\{X_{(k)}^{\mathscr{N}_{v}(t)}<z \mid \mathscr{N}_{v}(t) \geq k\right\} \\
= & \frac{\sum_{n=k}^{\infty} \operatorname{Pr}\left\{X_{(k)}^{\mathscr{N}_{v}(t)}<z, \mathscr{N}_{v}(t)=n\right\}}{\operatorname{Pr}\left\{\mathscr{N}_{v}(t) \geq k\right\}} \\
= & \frac{\sum_{n=k}^{\infty} \operatorname{Pr}\left\{X_{(k)}^{\mathscr{N}_{v}(t)}<z \mid \mathscr{N}_{v}(t)=n\right\} \operatorname{Pr}\left\{\mathscr{N}_{v}(t)=n\right\}}{\operatorname{Pr}\left\{\mathscr{N}_{v}(t) \geq k\right\}} .
\end{aligned}
$$


We can evaluate the numerator as

$$
\begin{aligned}
& \sum_{n=k}^{\infty} \operatorname{Pr}\left\{X_{(k)}^{\mathscr{N}_{v}(t)}<z \mid \mathscr{N}_{v}(t)=n\right\} \operatorname{Pr}\left\{\mathscr{N}_{v}(t)=n\right\} \\
& =\sum_{n=k}^{\infty}\left\{\sum_{j=k}^{n}\left(\begin{array}{l}
n \\
j
\end{array}\right) F^{j}(z)[1-F(z)]^{n-j}\right\} \frac{(-1)^{n}}{n !} \sum_{l=n}^{\infty} \frac{(-1)^{l}\left(\lambda t^{v}\right)^{l} l !}{(l-n) ! \Gamma(v l+1)} \\
& =\sum_{l=k}^{\infty} \frac{(-1)^{l}\left(\lambda t^{v}\right)^{l}}{\Gamma(v l+1)} \sum_{n=k}^{l}\left(\begin{array}{l}
l \\
n
\end{array}\right)(-1)^{n} \sum_{j=k}^{n}\left(\begin{array}{l}
n \\
j
\end{array}\right) F^{j}(z)[1-F(z)]^{n-j} \\
& =\sum_{l=k}^{\infty} \frac{(-1)^{l}\left(\lambda t^{v}\right)^{l}}{\Gamma(v l+1)} \sum_{j=k}^{l} F^{j}(z) \sum_{n=j}^{l}\left(\begin{array}{l}
l \\
n
\end{array}\right)\left(\begin{array}{l}
n \\
j
\end{array}\right)(-1)^{n}[1-F(z)]^{n-j} \\
& =\sum_{l=k}^{\infty} \frac{(-1)^{l}\left(\lambda t^{v}\right)^{l}}{\Gamma(v l+1)} \sum_{j=k}^{l} F^{j}(z)\left(\begin{array}{l}
l \\
j
\end{array}\right) \sum_{n=j}^{l}\left(\begin{array}{l}
l-j \\
n-j
\end{array}\right)(-1)^{n}[1-F(z)]^{n-j} \\
& =\sum_{l=k}^{\infty} \frac{(-1)^{l}\left(\lambda t^{v}\right)^{l}}{\Gamma(v l+1)} \sum_{j=k}^{l} F^{j}(z)\left(\begin{array}{l}
l \\
j
\end{array}\right) \sum_{r=0}^{l-j}\left(\begin{array}{c}
l-j \\
r
\end{array}\right)(-1)^{r+j}[1-F(z)]^{r} \\
& =\sum_{l=k}^{\infty} \frac{(-1)^{l}\left(\lambda t^{v}\right)^{l}}{\Gamma(v l+1)} \sum_{j=k}^{l} F^{j}(z)\left(\begin{array}{l}
l \\
j
\end{array}\right)(-1)^{j} F(z)^{l-j} \\
& =\sum_{l=k}^{\infty} \frac{(-1)^{l}\left(\lambda t^{v}\right)^{l}}{\Gamma(v l+1)} F^{l}(z) \sum_{j=k}^{l}\left(\begin{array}{l}
l \\
j
\end{array}\right)(-1)^{j} \\
& =\sum_{j=k}^{\infty} \sum_{l=j}^{\infty}\left(\begin{array}{l}
l \\
j
\end{array}\right)(-1)^{l+j} \frac{\left(F(z) \lambda t^{v}\right)^{l}}{\Gamma(v l+1)} \\
& =\sum_{j=k}^{\infty} \operatorname{Pr}\left\{\mathscr{N}_{v}^{\lambda F(z)}(t)=j\right\}=\operatorname{Pr}\left\{\mathscr{N}_{v}^{\lambda F(z)}(t) \geq k\right\} \text {. }
\end{aligned}
$$

By inserting (2.31) into (2.30) we get (2.28), in view of (2.10).

Remark 2.6 We note that for $z \rightarrow \infty$ the previous expression converges to 1 , as happens for the standard case (2.29).

As a particular case we can obtain the distribution of the minimum of a random number of i.i.d. random variables $X_{i}, i \geq 1$, by taking $k=1$ in (2.28):

$$
\operatorname{Pr}\left\{\min _{1 \leq j \leq \mathscr{N}_{v}(t)} X_{j}<z \mid \mathscr{N}_{v}(t) \geq 1\right\}=\frac{1-E_{v, 1}\left(-\lambda t^{v} F(z)\right)}{1-E_{v, 1}\left(-\lambda t^{v}\right)} .
$$

Again, for $z \rightarrow \infty$, (2.32) converges to 1 . 
As far as the distribution of the maximum is concerned we can evaluate it as follows:

$$
\begin{aligned}
& \operatorname{Pr}\left\{\max _{1 \leq j \leq \mathscr{N}_{v}(t)} X_{j}<z \mid \mathscr{N}_{v}(t) \geq 1\right\} \\
= & \sum_{n=1}^{\infty} F^{n}(z) \operatorname{Pr}\left\{\mathscr{N}_{v}(t)=n \mid \mathscr{N}_{v}(t) \geq 1\right\} \\
= & \frac{1}{\operatorname{Pr}\left\{\mathscr{N}_{v}(t) \geq 1\right\}} \sum_{n=1}^{\infty} \frac{(-1)^{n}}{n !} F^{n}(z) \sum_{l=n}^{\infty} \frac{(-1)^{l}\left(\lambda t^{v}\right)^{l} l !}{(l-n) ! \Gamma(v l+1)} \\
= & \frac{1}{\operatorname{Pr}\left\{\mathscr{N}_{v}(t) \geq 1\right\}} \sum_{l=1}^{\infty} \frac{(-1)^{l}\left(\lambda t^{v}\right)^{l}}{\Gamma(v l+1)} \sum_{n=1}^{l}\left(\begin{array}{l}
l \\
n
\end{array}\right)(-1)^{n} F^{n}(z) \\
= & \frac{1}{\operatorname{Pr}\left\{\mathscr{N}_{v}(t) \geq 1\right\}}\left\{\sum_{l=1}^{\infty} \frac{(-1)^{l}\left[\lambda t^{v}(1-F(z))\right]^{l}}{\Gamma(v l+1)}-\sum_{l=1}^{\infty} \frac{(-1)^{l}\left(\lambda t^{v}\right)^{l}}{\Gamma(v l+1)}\right\} \\
= & \frac{E_{v, 1}\left(-\lambda t^{v}(1-F(z))\right)-E_{v, 1}\left(-\lambda t^{v}\right)}{\operatorname{Pr}\left\{\mathscr{N}_{v}(t) \geq 1\right\}} \underset{z \rightarrow \infty}{\rightarrow} \frac{\operatorname{Pr}\left\{\mathscr{N}_{v}(t) \geq 1\right\}}{\operatorname{Pr}\left\{\mathscr{N}_{v}(t) \geq 1\right\}}=1 .
\end{aligned}
$$

We can verify that for $v=1$, i.e. in the standard case, result (2.33) yields the well known formula:

$$
\begin{aligned}
& \operatorname{Pr}\left\{\max _{1 \leq j \leq N(t)} X_{j}<z \mid N(t) \geq 1\right\} \\
= & \frac{e^{-\lambda t(1-F(z))}-e^{-\lambda t}}{1-e^{-\lambda t}} \rightarrow\left\{\begin{array}{cc}
0 & z \rightarrow 0 \\
1 & z \rightarrow \infty
\end{array} .\right.
\end{aligned}
$$

However if we consider that $\{N(t)=0\} \subset\left\{\max _{1 \leq j \leq N(t)} X_{j}<z\right\}=\Omega$, then

$$
\operatorname{Pr}\left\{\max _{1 \leq j \leq N(t)} X_{j}<z\right\}=\sum_{n=0}^{\infty} F(z)^{n} \operatorname{Pr}\{N(t)=n\}=e^{-\lambda t(1-F(z))}
$$

and this distribution has a jump of height $e^{-\lambda t}$ in $z=0$. If $N(t)=0$ we assume that $\max _{1 \leq j \leq N(t)} X_{j}=$ $-\infty$.

In the fractional case we have analogously that

$$
\begin{aligned}
& \operatorname{Pr}\left\{\max _{1 \leq j \leq \mathscr{N}_{v}(t)} X_{j}<z\right\} \\
= & \sum_{n=0}^{\infty} F(z)^{n} \frac{(-1)^{n}}{n !} \sum_{l=n}^{\infty} \frac{(-1)^{l}\left(\lambda t^{v}\right)^{l} l !}{(l-n) ! \Gamma(v l+1)} \\
= & \sum_{l=0}^{\infty} \frac{(-1)^{l}\left(\lambda t^{v}\right)^{l}}{\Gamma(v l+1)} \sum_{n=0}^{l}\left(\begin{array}{l}
l \\
n
\end{array}\right)(-1)^{n} F(z)^{n} \\
= & \sum_{l=0}^{\infty} \frac{\left(-\lambda t^{v}(1-F(z))\right)^{l}}{\Gamma(v l+1)}=E_{v, 1}\left(-\lambda t^{v}(1-F(z))\right) .
\end{aligned}
$$

The limit for $z \rightarrow \infty$ is 1 , while for $z \rightarrow 0$ there is a jump equal to $\operatorname{Pr}\left\{\mathscr{N}_{v}(t)=0\right\}$. 


\section{Planar random motions changing direction at fractional Poisson times}

Let us consider a planar random motion described by a particle moving at finite velocity $c$ and changing direction at Poisson time instants (for details on this process see Kolesnik and Orsingher (2005)). At each Poisson event, the new direction is chosen with uniform law in $[0,2 \pi]$. Let us denote the current position of the moving particle by $(X(t), Y(t)), t>0$. We recall the formulae (11) and (18) in Kolesnik and Orsingher (2005) that provide the conditional distribution of the planar random motion

$$
\begin{aligned}
& \operatorname{Pr}\{X(t) \in d x, Y(t) \in d y \mid N(t)=k\} \\
= & \left\{\begin{array}{l}
\delta\left(c^{2} t^{2}-\left(x^{2}+y^{2}\right)\right), \quad k=0 \\
\frac{k}{2 \pi(c t)^{k}}\left(c^{2} t^{2}-\left(x^{2}+y^{2}\right)\right)^{\frac{k}{2}-1} d x d y, \quad k \geq 1
\end{array}\right.
\end{aligned}
$$

for $t>0,(x, y) \in C_{c t}=\left\{x, y: \sqrt{x^{2}+y^{2}} \leq c t\right\}$ and the corresponding characteristic function

$$
\begin{aligned}
& \iint_{C_{c t}} e^{i \alpha x+i \beta y} \operatorname{Pr}\{X(t) \in d x, Y(t) \in d y \mid N(t)=k\} \\
= & \frac{2^{\frac{k}{2}} \Gamma\left(\frac{k}{2}+1\right)}{\left(c t \sqrt{\alpha^{2}+\beta^{2}}\right)^{\frac{k}{2}}} J_{\frac{k}{2}}\left(c t \sqrt{\alpha^{2}+\beta^{2}}\right), \quad k \geq 0,
\end{aligned}
$$

where $J_{\frac{k}{2}}$ is the Bessel function of order $k / 2$.

We will examine here the same planar motion (taken at a Brownian time) when the process governing the changes of direction is replaced by a fractional Poisson process. In particular, we will refer to the process $\mathscr{N}_{v}(t), t>0$ analyzed in Section 2 .

We start by considering the case where $v=\frac{1}{2}$, since it permits us to exploit the representation (2.23). Indeed in this case, in view of Theorem 2.1, the fractional process $\mathscr{N}_{\frac{1}{2}}$ coincides in distribution with a Poisson process at the random time $\mathscr{T}_{1}$. As we have already seen in Remark $2.1, \mathscr{T}_{1}$ reduces to the reflecting Brownian motion, i.e. $\mathscr{T}_{1}(t)=|B(t)|, t>0$, where again $B(t), t>0$ is a Brownian motion with infinitesimal variance 2 .

We present the distribution of $(X(|B(t)|), Y(|B(t)|)), t>0$, conditional on a fixed number of events $\mathscr{N}_{\frac{1}{2}}(t)=N(|B(t)|)=k, k \geq 0$. Clearly $B(t), t>0$ is independent from $(X(t), Y(t))$.

At time $|B(t)|$ the set of possible positions of the process is represented by the whole circle with radius $c|B(t)|$, if $k \geq 1$, and coincides with the circumference of the same circle, if $k=0$. In both cases, since the radius is the random variable $c|B(t)|$ distributed on the positive real line, the support covers the whole plane.

In the next theorem we will show that the distribution of the random vector under the condition of a number $k$ of events possesses a surprisingly explicit form: it coincides with a bivariate Gaussian with variance (itself random) equal to $2 c^{2} t W_{k}$, where $W_{k}$ is a Beta random variable with parameters $\frac{1}{2}, \frac{k+1}{2}$. 
Theorem 3.1 Under the condition $N_{\frac{1}{2}}(t)=k$ the conditional distribution of the vector $(X(|B(t)|), Y(|B(t)|))$ reads

$$
\begin{aligned}
& \operatorname{Pr}\left\{X(|B(t)|) \in d x, Y(|B(t)|) \in d y \mid \mathscr{N}_{\frac{1}{2}}(t)=k\right\} \\
= & \frac{d x d y}{B\left(\frac{1}{2}, \frac{k+1}{2}\right)} \int_{0}^{1} w^{-\frac{1}{2}}(1-w)^{\frac{k-1}{2}} \frac{e^{-\frac{x^{2}+y^{2}}{4 c^{2} t w}}}{4 \pi t w c^{2}} d w,
\end{aligned}
$$

for $k \geq 0,(x, y) \in \mathbb{R}^{2}$.

Proof For $k \geq 1$, in view of (3.1) we have that

$$
\begin{aligned}
& \operatorname{Pr}\left\{X(|B(t)|) \in d x, Y(|B(t)|) \in d y \mid \mathscr{N}_{\frac{1}{2}}(t)=k\right\} \\
= & \int_{0}^{\infty} \operatorname{Pr}\left\{X(|B(t)|) \in d x, Y(|B(t)|) \in d y\left|\mathscr{N}_{\frac{1}{2}}(t)=k,\right| B(t) \mid=s\right\} \operatorname{Pr}\{|B(t)| \in d s\} \\
= & {\left[\text { since } \mathscr{N}_{\frac{1}{2}}(t)=N(|B(t)|)\right] } \\
= & \int_{0}^{\infty} \operatorname{Pr}\{X(|B(t)|) \in d x, Y(|B(t)|) \in d y \mid N(s)=k\} \operatorname{Pr}\{|B(t)| \in d s\} \\
= & d x d y \int_{0}^{\infty} \frac{k}{2 \pi(c s)^{k}}\left(c^{2} s^{2}-\left(x^{2}+y^{2}\right)\right)^{\frac{k}{2}-1} 1_{\left\{x^{2}+y^{2} \leq c^{2} s^{2}\right\}} \frac{e^{-\frac{s^{2}}{4 t}}}{\sqrt{\pi t}} d s .
\end{aligned}
$$


We now take the Fourier transform of (3.4) and apply (3.2), so that we get

$$
\begin{aligned}
& \iint_{\mathbb{R}^{2}} e^{i \alpha x+i \beta y} d x d y \int_{0}^{\infty} \frac{k}{2 \pi(c s)^{k}}\left(c^{2} s^{2}-\left(x^{2}+y^{2}\right)\right)^{\frac{k}{2}-1} 1_{\left\{x^{2}+y^{2} \leq c^{2} s^{2}\right\}} \frac{e^{-\frac{s^{2}}{4 t}}}{\sqrt{\pi t}} d s \\
& =\int_{0}^{\infty} \frac{2^{\frac{k}{2}} \Gamma\left(\frac{k}{2}+1\right)}{\left(c s \sqrt{\alpha^{2}+\beta^{2}}\right)^{\frac{k}{2}}} J_{\frac{k}{2}}\left(\operatorname{cs} \sqrt{\alpha^{2}+\beta^{2}}\right) \frac{e^{-\frac{s^{2}}{4 t}}}{\sqrt{\pi t}} d s \\
& =\left[z=\frac{s^{2}}{4 t}\right] \\
& =\frac{2^{\frac{k}{2}} \Gamma\left(\frac{k}{2}+1\right)}{\left(c \sqrt{\alpha^{2}+\beta^{2}}\right)^{\frac{k}{2}}} \int_{0}^{\infty}(\sqrt{4 t z})^{-\frac{k}{2}} J_{\frac{k}{2}}\left(c \sqrt{4 t z} \sqrt{\alpha^{2}+\beta^{2}}\right) \frac{e^{-z}}{\sqrt{\pi z}} d z \\
& =\frac{t^{-\frac{k}{4}} \Gamma\left(\frac{k}{2}+1\right)}{\sqrt{\pi}\left(c \sqrt{\alpha^{2}+\beta^{2}}\right)^{\frac{k}{2}}} \sum_{m=0}^{\infty} \frac{(-1)^{m}}{m ! \Gamma\left(m+\frac{k}{2}+1\right)}\left(\frac{c \sqrt{4 t} \sqrt{\alpha^{2}+\beta^{2}}}{2}\right)^{2 m+\frac{k}{2}} \times \\
& \times \int_{0}^{\infty} z^{m+\frac{k}{4}-\frac{k}{4}-\frac{1}{2}} e^{-z} d z \\
& =\frac{t^{-\frac{k}{4}} \Gamma\left(\frac{k}{2}+1\right)}{\sqrt{\pi}\left(c \sqrt{\alpha^{2}+\beta^{2}}\right)^{\frac{k}{2}}} \sum_{m=0}^{\infty} \frac{(-1)^{m} \Gamma\left(m+\frac{1}{2}\right)}{m ! \Gamma\left(m+\frac{k}{2}+1\right)}\left(c \sqrt{t} \sqrt{\alpha^{2}+\beta^{2}}\right)^{2 m+\frac{k}{2}} \\
& =\frac{t^{-\frac{k}{4}} \Gamma\left(\frac{k}{2}+1\right)}{\sqrt{\pi}\left(c \sqrt{\alpha^{2}+\beta^{2}}\right)^{\frac{k}{2}}} \sum_{m=0}^{\infty}(-1)^{m} \frac{\left(c \sqrt{t} \sqrt{\alpha^{2}+\beta^{2}}\right)^{2 m+\frac{k}{2}}}{m ! \Gamma\left(\frac{k}{2}+\frac{1}{2}\right)} B\left(\frac{k}{2}+\frac{1}{2}, m+\frac{1}{2}\right) \\
& =\frac{\Gamma\left(\frac{k}{2}+1\right)}{\Gamma\left(\frac{1}{2}\right) \Gamma\left(\frac{k}{2}+\frac{1}{2}\right)} \int_{0}^{1} w^{-\frac{1}{2}}(1-w)^{\frac{k}{2}-\frac{1}{2}} \sum_{m=0}^{\infty}(-1)^{m} \frac{\left(c^{2} t w\left(\alpha^{2}+\beta^{2}\right)\right)^{m}}{m !} d w \\
& =\frac{\Gamma\left(\frac{k}{2}+1\right)}{\Gamma\left(\frac{1}{2}\right) \Gamma\left(\frac{k}{2}+\frac{1}{2}\right)} \int_{0}^{1} w^{-\frac{1}{2}}(1-w)^{\frac{k}{2}-\frac{1}{2}} e^{-c^{2} t w\left(\alpha^{2}+\beta^{2}\right)} d w \\
& =\frac{1}{B\left(\frac{1}{2}, \frac{k}{2}+\frac{1}{2}\right)} \int_{0}^{1} w^{-\frac{1}{2}}(1-w)^{\frac{k}{2}-\frac{1}{2}} e^{-c^{2} t w\left(\alpha^{2}+\beta^{2}\right)} d w .
\end{aligned}
$$

By inverting the characteristic function (3.5) we get formula (3.3), for $k \geq 1$.

For $k=0$, by performing similar steps we can check that result (3.3) is valid also in this case.

We can analogously consider the planar random motion changing direction at fractional Poisson times of order $v=\frac{1}{4}$, by applying the results obtained in Orsingher and Beghin (2009). In this paper it is proved that the solution to the fractional diffusion equation of order $\frac{1}{2^{n}}$

$$
\left\{\begin{array}{l}
\frac{\partial \frac{1}{2^{n}} v}{\partial t^{\frac{1}{2^{n}}}}=2^{\frac{1}{2^{n}}}-2 \frac{\partial^{2} v}{\partial x^{2}} \\
v(x, 0)=\delta(x)
\end{array}\right.
$$


can be written as

$$
v_{\frac{1}{2^{n}}}(x, t)=2^{n} \int_{0}^{\infty} \cdots \int_{0}^{\infty} \frac{e^{-\frac{x^{2}}{2 z_{1}}}}{\sqrt{2 \pi z_{1}}} \frac{e^{-\frac{z_{1}^{2}}{2 z_{2}}}}{\sqrt{2 \pi z_{2}}} \cdots \frac{e^{-\frac{z_{n}^{2}}{2 t}}}{\sqrt{2 \pi t}} d z_{1} \cdots d z_{n} .
$$

Formula (3.7) coincides with the density of the $n$-times iterated Brownian motion

$$
\mathscr{I}_{n}(t)=\mathscr{B}_{1}\left(\left|\mathscr{B}_{2}\left(\left|\mathscr{B}_{3}\left(\cdots\left(\left|\mathscr{B}_{n+1}(t)\right|\right) \cdots\right)\right|\right)\right|\right), \quad t>0,
$$

where the $\mathscr{B}_{j}$ 's are standard independent Brownian motions (i.e. with infinitesimal variance 1).

In order to apply Theorem 2.1, we must suitably adapt the previous results, taking into account that, for $v=\frac{1}{4}$, equation $(2.12)$ reduces to

$$
\left\{\begin{array}{l}
\frac{\partial^{\frac{1}{2}} v}{\partial t^{\frac{1}{2}}}=\frac{\partial^{2} v}{\partial x^{2}}, \quad t>0, x \in \mathbb{R} \\
v(x, 0)=\delta(x)
\end{array} \quad t\right.
$$

and this differs from (3.6) for the coefficient representing the infinitesimal variance. Thus, instead of (3.7), we must use the solution to (3.9), which reads

$$
v_{\frac{1}{2}}(x, t)=2 \int_{0}^{\infty} \frac{e^{-\frac{x^{2}}{4 s}}}{\sqrt{4 \pi s}} \frac{e^{-\frac{s^{2}}{4 t}}}{\sqrt{4 \pi t}} d s, \quad t>0, x \in \mathbb{R} .
$$

By folding (3.10) we get

$$
\bar{v}_{\frac{1}{2}}(x, t)=\left\{\begin{array}{l}
2 v_{\frac{1}{2}}(x, t), \quad x>0 \\
0, \quad x<0
\end{array} .\right.
$$

Therefore we can interpret the random time as the iterated reflected Brownian motion $\mathscr{T}_{2}(t)=$ $\left|I_{1}(t)\right|=\left|B_{1}\left(\left|B_{2}(t)\right|\right)\right|$, where $B_{1}$ and $B_{2}$ are independent Brownian motions with infinitesimal variance 2 . We study the random motion under the condition that the changes of directions are governed by a fractional Poisson process $\mathscr{N}_{\frac{1}{4}}(t)=N\left(\left|B_{1}\left(\left|B_{2}(t)\right|\right)\right|\right)=k, k \geq 0$.

Theorem 3.2 Under the condition $\mathscr{N}_{\frac{1}{4}}(t)=k$, the conditional distribution of the vector $\left(X\left(\left|B_{1}\left(\left|B_{2}(t)\right|\right)\right|\right), Y\left(\left|B_{1}\left(\left|B_{2}(t)\right|\right)\right|\right)\right)$ reads

$$
\begin{aligned}
& \operatorname{Pr}\left\{X\left(\left|B_{1}\left(\left|B_{2}(t)\right|\right)\right|\right) \in d x, Y\left(\left|B_{1}\left(\left|B_{2}(t)\right|\right)\right|\right) \in d y \mid \mathscr{N}_{\frac{1}{4}}(t)=k\right\} \\
= & \frac{d x d y}{B\left(\frac{1}{2}, \frac{k}{2}+\frac{1}{2}\right)} \int_{0}^{\infty} \frac{e^{-\frac{z^{2}}{4 t}}}{\sqrt{\pi t}} d z \int_{0}^{1} w^{-\frac{1}{2}}(1-w)^{\frac{k}{2}-\frac{1}{2}} \frac{e^{-\frac{x^{2}+y^{2}}{4 c^{2} z w}}}{4 \pi c^{2} z w} d w
\end{aligned}
$$

for $k \geq 0,(x, y) \in \mathbb{R}^{2}$. 
Proof As in (3.4) we get, for $k \geq 1$,

$$
\begin{aligned}
& \operatorname{Pr}\left\{X\left(\left|B_{1}\left(\left|B_{2}(t)\right|\right)\right|\right) \in d x, Y\left(\left|B_{1}\left(\left|B_{2}(t)\right|\right)\right|\right) \in d y \mid \mathscr{N}_{\frac{1}{4}}(t)=k\right\} \\
= & \int_{0}^{\infty} \operatorname{Pr}\left\{X\left(\left|B_{1}\left(\left|B_{2}(t)\right|\right)\right|\right) \in d x, Y\left(\left|B_{1}\left(\left|B_{2}(t)\right|\right)\right|\right) \in d y \mid N(s)=k\right\} \operatorname{Pr}\left\{\left(\left|B_{1}\left(\left|B_{2}(t)\right|\right)\right|\right) \in d s\right\} \\
= & d x d y \int_{0}^{\infty} \int_{0}^{\infty} \frac{k}{2 \pi(c s)^{k}}\left(c^{2} s^{2}-\left(x^{2}+y^{2}\right)\right)^{\frac{k}{2}-1} 1_{\left\{x^{2}+y^{2} \leq c^{2} s^{2}\right\}} \frac{e^{-\frac{s^{2}}{4 z}}}{\sqrt{\pi z}} \frac{e^{-\frac{z^{2}}{4 t}}}{\sqrt{\pi t}} d z d s .
\end{aligned}
$$

By taking the Fourier transform of (3.12) we get

$$
\begin{aligned}
& \iint_{\mathbb{R}^{2}} e^{i \alpha x+i \beta y} d x d y \int_{0}^{\infty} \int_{0}^{\infty} \frac{k}{2 \pi(c s)^{k}}\left(c^{2} s^{2}-\left(x^{2}+y^{2}\right)\right)^{\frac{k}{2}-1} 1_{\left\{x^{2}+y^{2} \leq c^{2} s^{2}\right\}} \\
& \cdot \frac{e^{-\frac{s^{2}}{4 z}}}{\sqrt{\pi z}} \frac{e^{-\frac{z^{2}}{4 t}}}{\sqrt{\pi t}} d z d s \\
= & \int_{0}^{\infty} \int_{0}^{\infty} \frac{2^{\frac{k}{2}} \Gamma\left(\frac{k}{2}+1\right)}{\left(c s \sqrt{\alpha^{2}+\beta^{2}}\right)^{\frac{k}{2}}} J_{\frac{k}{2}}\left(c s \sqrt{\alpha^{2}+\beta^{2}}\right) \frac{e^{-\frac{s^{2}}{4 z}}}{\sqrt{\pi z}} \frac{e^{-\frac{z^{2}}{4 t}}}{\sqrt{\pi t}} d z d s \\
= & {\left[w=\frac{s^{2}}{4 z}\right] } \\
= & \frac{2^{\frac{k}{2}} \Gamma\left(\frac{k}{2}+1\right)}{\left(c \sqrt{\alpha^{2}+\beta^{2}}\right)^{\frac{k}{2}}} \int_{0}^{\infty} \int_{0}^{\infty}(\sqrt{4 z w})^{-\frac{k}{2}} J_{\frac{k}{2}}\left(c \sqrt{4 z w} \sqrt{\alpha^{2}+\beta^{2}}\right) \frac{e^{-w}}{\sqrt{\pi w}} \frac{e^{-\frac{z^{2}}{4 t}}}{\sqrt{\pi t}} d w d z \\
= & \frac{\Gamma\left(\frac{k}{2}+1\right)}{\left(c \sqrt{\alpha^{2}+\beta^{2}}\right)^{\frac{k}{2}}} \int_{0}^{\infty} z^{-\frac{k}{4}} \frac{e^{-\frac{z^{2}}{4 t}}}{\sqrt{\pi t}} \sum_{m=0}^{\infty} \frac{(-1)^{m}}{m ! \Gamma\left(m+\frac{k}{2}+1\right)}\left(\frac{c \sqrt{4 z} \sqrt{\alpha^{2}+\beta^{2}}}{2}\right)^{2 m+\frac{k}{2}} d z . \\
& \int_{0}^{\infty} w^{m+\frac{k}{4}-\frac{k}{4}-\frac{1}{2}} e^{-w} d w \\
= & \frac{\Gamma\left(\frac{k}{2}+1\right)}{\Gamma\left(\frac{1}{2}\right)\left(c \sqrt{\alpha^{2}+\beta^{2}}\right)^{\frac{k}{2}}} \int_{0}^{\infty} z^{-\frac{k}{4}} \frac{e^{-\frac{z^{2}}{4 t}}}{\sqrt{\pi t}} d z \sum_{m=0}^{\infty}(-1)^{m} \frac{\left(c \sqrt{z} \sqrt{\alpha^{2}+\beta^{2}}\right)^{2 m+\frac{k}{2}}}{m ! \Gamma\left(\frac{k}{2}+\frac{1}{2}\right)} B\left(\frac{k}{2}+\frac{1}{2}, m+\frac{1}{2}\right)
\end{aligned}
$$

so that we derive (3.11).

We can conclude that the random vector, under the condition of a number $k$ of Poisson events, possesses a bivariate Gaussian distribution with variance (itself random) equal to $2 c^{2}|B(t)| W_{k}$, where $W_{k}$ is a Beta random variable with parameters $\frac{1}{2}, \frac{k+1}{2}$ and $B(t)$ is a Brownian motion with infinitesimal variance 2 .

Remark 3.1 For $v=\frac{1}{2^{n}}, n>2$, we can generalize the previous results: the fractional Poisson process is, in this case, represented by $\mathscr{N}_{\frac{1}{2^{n}}}(t)=N\left(\mathscr{T}_{\frac{1}{2^{n-1}}}(t)\right), t>0$ and the random time is $\mathscr{T}_{\frac{1}{2^{n-1}}}(t)=$ 
$\left|I_{n-1}(t)\right|=\left|B_{1}\left(\ldots\left|B_{n}(t)\right| \ldots\right)\right|$. Thus we obtain, by steps similar to those of Theorems 3.1 and 3.2, the following conditional distribution for the planar random vector:

$$
\begin{aligned}
& \operatorname{Pr}\left\{X\left(\mid B_{1}\left(\ldots\left|B_{n}(t)\right| \ldots \mid\right)\right) \in d x, Y\left(\mid B_{1}\left(\ldots\left|B_{n}(t)\right| \ldots \mid\right)\right) \in d y \mid \mathscr{N}_{\frac{1}{2^{n}}}(t)=k\right\} \\
= & \frac{d x d y}{B\left(\frac{1}{2}, \frac{k}{2}+\frac{1}{2}\right)} \int_{0}^{\infty} \frac{e^{-\frac{z_{1}^{2}}{4 z_{2}}}}{\sqrt{\pi z_{2}}} d z_{1} \ldots \int_{0}^{\infty} \frac{e^{-\frac{z_{n-1}^{2}}{4 t}}}{\sqrt{\pi t}} d z_{n-1} \int_{0}^{1} w^{-\frac{1}{2}}(1-w)^{\frac{k}{2}-\frac{1}{2}} \frac{e^{-\frac{x^{2}+y^{2}}{4 c^{2} z_{1} w}}}{4 \pi c^{2} z_{1} w} d w,
\end{aligned}
$$

for $k \geq 0$ and thus the process possesses a conditional distribution coinciding with the bivariate Gaussian law with variance equal to $2 c^{2}\left|B_{1}\left(\ldots\left|B_{n-1}(t)\right| \ldots\right)\right| W_{k}$. It is easy to check that in the case $v=\frac{1}{4}$, i.e. for $n=2$, formula (3.14) reduces to (3.11).

We consider now the general case $0<v \leq 1$ : the random process representing the time argument is $\mathscr{T}_{2 v}(t), t>0$ and the condition is therefore equal to $\mathscr{N}_{v}(t)=N\left(\mathscr{T}_{2 v}(t)\right)=k$.

Theorem 3.3 Under the condition $\mathscr{N}_{v}(t)=k$, the conditional characteristic function of the vector $\left(X\left(\mathscr{T}_{2 v}(t)\right), Y\left(\mathscr{T}_{2 v}(t)\right)\right)$ reads

$$
\begin{aligned}
& \iint_{\mathbb{R}^{2}} e^{i \alpha x+i \beta y} \operatorname{Pr}\left\{X\left(\mathscr{T}_{2 v}(t)\right) \in d x, Y\left(\mathscr{T}_{2 v}(t)\right) \in d y \mid N\left(\mathscr{T}_{2 v}(t)\right)=k\right\} \\
= & \frac{d x d y}{B\left(\frac{1}{2}, \frac{k+1}{2}\right)} \int_{0}^{1} w^{-\frac{1}{2}}(1-w)^{\frac{k-1}{2}} E_{2 v, 1}\left(-w t^{2 v} c^{2}\left(\alpha^{2}+\beta^{2}\right)\right) d w .
\end{aligned}
$$

Proof In this case we must evaluate the following integral (which is analogous to (3.5) and (3.13)) 


$$
\begin{aligned}
& \frac{2^{\frac{k}{2}} \Gamma\left(\frac{k}{2}+1\right)}{\left(c \sqrt{\alpha^{2}+\beta^{2}}\right)^{\frac{k}{2}}} \int_{0}^{\infty} s^{-\frac{k}{2}} J_{\frac{k}{2}}\left(c s \sqrt{\alpha^{2}+\beta^{2}}\right) \frac{1}{t^{v}} W_{-v, 1-v}\left(-\frac{s}{t^{v}}\right) d s \\
& =\text { by (2.24) ] } \\
& =\frac{2^{\frac{k}{2}} \Gamma\left(\frac{k}{2}+1\right)}{\left(c \sqrt{\alpha^{2}+\beta^{2}}\right)^{\frac{k}{2}} t^{v}} \frac{1}{2 \pi i} \int_{H a} \frac{e^{z}}{z^{1-v}} d z \int_{0}^{\infty} s^{-\frac{k}{2}} J_{\frac{k}{2}}\left(c s \sqrt{\alpha^{2}+\beta^{2}}\right) e^{-s \frac{z^{v}}{t^{v}}} d s \\
& =\frac{2^{\frac{k}{2}} \Gamma\left(\frac{k}{2}+1\right)}{\left(c \sqrt{\alpha^{2}+\beta^{2}}\right)^{\frac{k}{2}} t^{v}} \frac{1}{2 \pi i} \int_{H a} \frac{e^{z}}{z^{1-v}} d z \times \\
& \times \int_{0}^{\infty} s^{-\frac{k}{2}} \sum_{m=0}^{\infty}\left(\frac{c s \sqrt{\alpha^{2}+\beta^{2}}}{2}\right)^{2 m+\frac{k}{2}} \frac{(-1)^{m}}{m ! \Gamma\left(m+\frac{k}{2}+1\right)} e^{-s \frac{z^{v}}{t^{v}}} d s \\
& =\frac{\Gamma\left(\frac{k}{2}+1\right)}{2 \pi i} \int_{H a} \frac{e^{z}}{z} \sum_{m=0}^{\infty}\left(\frac{c t^{v} \sqrt{\alpha^{2}+\beta^{2}}}{2 z^{v}}\right)^{2 m} \frac{(-1)^{m} \Gamma(2 m+1)}{m ! \Gamma\left(m+\frac{k}{2}+1\right)} d z \\
& =\quad \text { by }(2.26)] \\
& =\Gamma\left(\frac{k}{2}+1\right) \sum_{m=0}^{\infty}\left(\frac{c t^{v} \sqrt{\alpha^{2}+\beta^{2}}}{2}\right)^{2 m} \frac{(-1)^{m} \Gamma(2 m+1)}{\Gamma(2 m v+1) m ! \Gamma\left(m+\frac{k}{2}+1\right)} \\
& =\frac{\Gamma\left(\frac{k}{2}+1\right)}{\Gamma\left(\frac{k+1}{2}\right)} \sum_{m=0}^{\infty}(-1)^{m}\left(\frac{c t^{v} \sqrt{\alpha^{2}+\beta^{2}}}{2}\right)^{2 m} \frac{\Gamma(2 m+1) \Gamma\left(m+\frac{1}{2}\right) \Gamma\left(\frac{k+1}{2}\right)}{\Gamma(2 m v+1) m ! \Gamma\left(m+\frac{1}{2}\right) \Gamma\left(m+\frac{k}{2}+1\right)} \\
& =\frac{\Gamma\left(\frac{k}{2}+1\right)}{\Gamma\left(\frac{k+1}{2}\right)} \int_{0}^{1} w^{-\frac{1}{2}}(1-w)^{\frac{k-1}{2}} \sum_{m=0}^{\infty}(-1)^{m}\left(\frac{c t^{v} \sqrt{\alpha^{2}+\beta^{2}} \sqrt{w}}{2}\right)^{2 m} \frac{\Gamma(2 m+1) d w}{\Gamma(2 m v+1) m ! \Gamma\left(m+\frac{1}{2}\right)} \\
& =\frac{1}{B\left(\frac{1}{2}, \frac{k+1}{2}\right)} \int_{0}^{1} w^{-\frac{1}{2}}(1-w)^{\frac{k-1}{2}} \sum_{m=0}^{\infty}\left(-\frac{c^{2} t^{2 v} w\left(\alpha^{2}+\beta^{2}\right)}{2^{2}}\right)^{m} \frac{\Gamma(2 m+1) \Gamma(m) d w}{\Gamma(2 m v+1) m ! 2^{1-2 m} \Gamma(2 m)} \\
& =\frac{1}{B\left(\frac{1}{2}, \frac{k+1}{2}\right)} \int_{0}^{1} w^{-\frac{1}{2}}(1-w)^{\frac{k-1}{2}} \sum_{m=0}^{\infty}\left(-c^{2} t^{2 v} w\left(\alpha^{2}+\beta^{2}\right)\right)^{m} \frac{d w}{\Gamma(2 m v+1)},
\end{aligned}
$$

from which we derive (3.15).

We note that, for $v=\frac{1}{2}$, formula (3.15) reduces to (3.5).

Theorems 3.1, 3.2 and 3.3, as well as Remark 3.1, show that the assumption of random time for the planar motion at finite velocity (with direction changing at fractional Poisson times) implies a drastic change in its structure. This is transparent in (3.5) where the resulting motion is a planar Brownian motion, whose infinitesimal variance is $2 c^{2} W_{k}$ and $W_{k} \sim \operatorname{Beta}\left(\frac{1}{2}, \frac{k+1}{2}\right)$. Therefore the larger the number $k$ of changes of direction, the more concentrated becomes the distribution of the random position. Clearly $\mathbb{E} W_{k}=\frac{1}{k+2}$ and this should convince the reader that the planar motion considered has sample paths that coil up around the starting point.

We now derive the law of the planar random motion in the general case $0<v \leq 1$. We will see 
that it can be expressed explicitly in terms of the planar process governed by the following twodimensional fractional differential equation:

$$
\left\{\begin{array}{l}
\frac{\partial^{2 v} u}{\partial t^{2 v}}=\lambda^{2}\left\{\frac{\partial^{2}}{\partial x^{2}}+\frac{\partial^{2}}{\partial y^{2}}\right\} u \\
u(x, y, 0)=\delta(x) \delta(y)
\end{array} \quad, 0<v \leq 1\right.
$$

with the additional condition

$$
u_{t}(x, y, 0)=0, \quad \text { for } \frac{1}{2}<v \leq 1
$$

Also in this case the time argument of the solution $u_{2 v}=u_{2 v}\left(x, y, t ; \lambda^{2}\right)$ will be itself random and will be represented in terms of a r.v. $W_{k} \sim \operatorname{Beta}\left(\frac{1}{2}, \frac{k+1}{2}\right)$.

We note that equation (3.16) includes, as particular cases, the planar wave equation (for $v=1$ ) and the planar heat-equation (for $v=\frac{1}{2}$ ).

Theorem 3.4 Under the condition $\mathscr{N}_{v}(t)=k$, the conditional distribution of the vector $\left(X\left(\mathscr{T}_{2 v}(t)\right), Y\left(\mathscr{T}_{2 v}(t)\right)\right), t>0$ reads

$$
\begin{aligned}
& \operatorname{Pr}\left\{X\left(\mathscr{T}_{2 v}(t)\right) \in d x, Y\left(\mathscr{T}_{2 v}(t)\right) \in d y \mid N\left(\mathscr{T}_{2 v}(t)\right)=k\right\} \\
= & \frac{d x d y}{B\left(\frac{1}{2}, \frac{k+1}{2}\right)} \int_{0}^{1} w^{-\frac{1}{2}}(1-w)^{\frac{k-1}{2}} \frac{d w}{2 \pi} \int_{0}^{\infty} \rho J_{0}\left(\rho \sqrt{x^{2}+y^{2}}\right) E_{2 v, 1}\left(-w t^{2 v} c^{2} \rho^{2}\right) d \rho \\
= & \frac{d x d y}{B\left(\frac{1}{2}, \frac{k+1}{2}\right)} \int_{0}^{1} w^{-\frac{1}{2}}(1-w)^{\frac{k-1}{2}} u_{2 v}\left(x, y, t ; c^{2} w\right) d w,
\end{aligned}
$$

where $u_{2 v}\left(x, y, t ; c^{2} w\right)$ is the solution to the fractional two-dimensional heat-wave equation (3.16), where $\lambda^{2}=c^{2} w$.

Proof The inverse Fourier transform of $E_{2 v, 1}\left(-w t^{2 v} c^{2}\left(\alpha^{2}+\beta^{2}\right)\right)$ can be calculated as follows

$$
\begin{aligned}
& \frac{1}{(2 \pi)^{2}} \iint_{\mathbb{R}^{2}} e^{-i \alpha x-i \beta y} E_{2 v, 1}\left(-w t^{2 v} c^{2}\left(\alpha^{2}+\beta^{2}\right)\right) d \alpha d \beta \\
= & \frac{1}{(2 \pi)^{2}} \int_{0}^{\infty} \rho d \rho \int_{0}^{2 \pi} e^{-i \rho(x \cos \theta+y \sin \theta)} E_{2 v, 1}\left(-w t^{2 v} c^{2} \rho^{2}\right) d \theta \\
= & \frac{1}{2 \pi} \int_{0}^{\infty} \rho J_{0}\left(\rho \sqrt{x^{2}+y^{2}}\right) E_{2 v, 1}\left(-w t^{2 v} c^{2} \rho^{2}\right) d \rho,
\end{aligned}
$$

so that we get the first form of (3.18). In (3.19) we used the following integral representation of the Bessel function:

$$
J_{0}\left(\rho \sqrt{x^{2}+y^{2}}\right)=\frac{1}{2 \pi} \int_{0}^{2 \pi} e^{-i \rho(x \cos \theta+y \sin \theta)} d \theta .
$$

We observe that (3.19) coincides with the solution $u_{2 v}\left(x, y, t ; \lambda^{2}\right)$ of the fractional equation (3.16), for $\lambda^{2}=c^{2} w$, and then the second form of (3.18) follows. 
Remark 3.2 As a check we put $v=\frac{1}{2}$, so that formula (3.19) reduces to the bivariate Gaussian density

$$
\begin{aligned}
& u_{1}\left(x, y, t ; c^{2} w\right)=\frac{1}{2 \pi} \int_{0}^{\infty} \rho J_{0}\left(\rho \sqrt{x^{2}+y^{2}}\right) e^{-w t c^{2} \rho^{2}} d \rho \\
= & {\left[\rho^{\prime}=w t c^{2} \rho^{2}\right] } \\
= & \frac{1}{2 \pi} \sum_{k=0}^{\infty}(-1)^{k}\left(\frac{\sqrt{x^{2}+y^{2}}}{2}\right)^{2 k} \frac{1}{k !^{2}} \int_{0}^{\infty} e^{-\rho} \frac{\rho^{k+\frac{1}{2}}}{\left(w t c^{2}\right)^{k+1}} \frac{1}{2 \sqrt{\rho}} d \rho \\
= & \frac{1}{4 \pi w t c^{2}} \sum_{k=0}^{\infty}(-1)^{k}\left(\frac{x^{2}+y^{2}}{4 w t c^{2}}\right)^{k} \frac{1}{k !}=\frac{e^{-\frac{x^{2}+y^{2}}{4 w t c^{2}}}}{4 \pi w t c^{2}},
\end{aligned}
$$

which, inserted into (3.18), gives (3.3).

For $v=1$, formula (3.19), by considering that $E_{2,1}(-x)=\cos (\sqrt{x})$, becomes

$$
\begin{aligned}
u_{2}\left(x, y, t ; c^{2} w\right) & =\frac{1}{2 \pi} \int_{0}^{\infty} \rho J_{0}\left(\rho \sqrt{x^{2}+y^{2}}\right) \cos (c \sqrt{w} t \rho) d \rho \\
& =\frac{1}{2 \pi c \sqrt{w}} \frac{\partial}{\partial t} \frac{1_{C_{c t \sqrt{w}}}(x, y)}{\sqrt{c^{2} t^{2} w-\left(x^{2}+y^{2}\right)}}
\end{aligned}
$$

which coincides with the fundamental solution to the planar wave equation (3.16) with the initial condition (3.17), for $\lambda^{2}=c^{2} w$ (see Smirnov (1981), p.581, and Orsingher (1985)).

We can show that the two expressions in (3.21) coincide by evaluating the Fourier transform of the second line:

$$
\begin{aligned}
& \frac{1}{2 \pi c \sqrt{w}} \frac{\partial}{\partial t} \iint_{\mathbb{R}^{2}} \frac{e^{i \alpha x+i \beta y} 1_{C_{c t \sqrt{w}}}(x, y) d x d y}{\sqrt{c^{2} t^{2} w-\left(x^{2}+y^{2}\right)}} \\
= & \frac{1}{2 \pi c \sqrt{w}} \frac{\partial}{\partial t} \int_{0}^{2 \pi} \int_{0}^{c t \sqrt{w}} \frac{\rho e^{i \rho(\alpha \cos \theta+\beta \sin \theta)} d \theta d \rho}{\sqrt{c^{2} t^{2} w-\rho^{2}}} \\
= & \frac{1}{c \sqrt{w}} \frac{\partial}{\partial t} \int_{0}^{c t \sqrt{w}} \frac{\rho J_{0}\left(\rho \sqrt{\alpha^{2}+\beta^{2}}\right) d \rho}{\sqrt{c^{2} t^{2} w-\rho^{2}}} \\
= & {[\text { by the change of variable } \rho=c t \sqrt{w} z] } \\
= & \frac{\partial}{\partial t} \int_{0}^{1} \frac{z t J_{0}\left(c t z \sqrt{w} \sqrt{\alpha^{2}+\beta^{2}}\right) d z}{\sqrt{1-z^{2}}} .
\end{aligned}
$$


The characteristic function (3.22) can be inverted as follows

$$
\begin{aligned}
& \frac{1}{(2 \pi)^{2}} \iint_{\mathbb{R}^{2}} e^{-i \alpha x-i \beta y} \frac{\partial}{\partial t} \int_{0}^{1} \frac{z t J_{0}\left(c t z \sqrt{w} \sqrt{\alpha^{2}+\beta^{2}}\right) d z}{\sqrt{1-z^{2}}} d \alpha d \beta, \\
= & \frac{1}{2 \pi} \int_{0}^{\infty} \rho J_{0}\left(\rho \sqrt{x^{2}+y^{2}}\right) d \rho \frac{\partial}{\partial t} \int_{0}^{1} \frac{z t J_{0}(c t z \sqrt{w} \rho) d z}{\sqrt{1-z^{2}}} \\
= & \frac{1}{2 \pi} \int_{0}^{\infty} \rho J_{0}\left(\rho \sqrt{x^{2}+y^{2}}\right) d \rho \frac{\partial}{\partial t}\left[t \sum_{m=0}^{\infty} \frac{(-1)^{m}}{m !^{2}}\left(\frac{c t z \sqrt{w} \rho}{2}\right)^{2 m} \int_{0}^{1} \frac{z^{2 m+1} d z}{\sqrt{1-z^{2}}}\right] \\
= & \frac{1}{2 \pi} \int_{0}^{\infty} \rho J_{0}\left(\rho \sqrt{x^{2}+y^{2}}\right) \frac{\partial}{\partial t}\left[\frac{1}{c \sqrt{w} \rho} \sin (c t \sqrt{w} \rho)\right] d \rho
\end{aligned}
$$

which coincides with the first line of (3.21).

We consider now the unconditional distribution of $\left(X\left(\mathscr{T}_{2 v}(t)\right), Y\left(\mathscr{T}_{2 v}(t)\right)\right), t>0$, that, for any $v \in(0,1]$, is presented in the next result:

Theorem 3.5 The distribution of $\left(X\left(\mathscr{T}_{2 v}(t)\right), Y\left(\mathscr{T}_{2 v}(t)\right)\right), t>0$ has the following representation

$$
\begin{aligned}
& \quad \operatorname{Pr}\left\{X\left(\mathscr{T}_{2 v}(t)\right) \in d x, Y\left(\mathscr{T}_{2 v}(t)\right) \in d y\right\} \\
& =\frac{d x d y}{\pi^{2}} \int_{0}^{1} \frac{u_{2 v}\left(x, y, t ; c^{2} w\right)}{w^{1 / 2}} d w \int_{0}^{1} \frac{d z}{\sqrt{z(1-z)}} \times \\
& \times\left[\frac{E_{v, 1}\left(-\lambda t^{v}\right)}{\sqrt{1-w}}+\frac{\pi \lambda t^{v}}{2 v} E_{v, v}\left(-\lambda t^{v}(1-\sqrt{(1-w)(1-z)})\right)\right],
\end{aligned}
$$

for $v \in(0,1]$.

Proof In view of formulae (2.10) and (3.18) we have that

$$
\begin{aligned}
& \operatorname{Pr}\left\{X\left(\mathscr{T}_{2 v}(t)\right) \in d x, Y\left(\mathscr{T}_{2 v}(t)\right) \in d y\right\} \\
= & \sum_{k=0}^{\infty} \operatorname{Pr}\left\{X\left(\mathscr{T}_{2 v}(t)\right) \in d x, Y\left(\mathscr{T}_{2 v}(t)\right) \in d y \mid N\left(\mathscr{T}_{2 v}(t)\right)=k\right\} \operatorname{Pr}\left\{N\left(\mathscr{T}_{2 v}(t)\right)=k\right\} \\
= & d x d y \int_{0}^{1} w^{-\frac{1}{2}}(1-w)^{-\frac{1}{2}} u_{2 v}\left(x, y, t ; c^{2} w\right) d w \sum_{k=0}^{\infty} \frac{(1-w)^{k / 2}}{B\left(\frac{1}{2}, \frac{k+1}{2}\right)} \sum_{r=k}^{\infty}(-1)^{r+k}\left(\begin{array}{l}
r \\
k
\end{array}\right) \frac{\left(\lambda t^{v}\right)^{r}}{\Gamma(v r+1)} .
\end{aligned}
$$

The double sum appearing in (3.24) can be further treated as follows:

$$
\begin{aligned}
& \sum_{k=0}^{\infty} \frac{(1-w)^{k / 2} \Gamma\left(\frac{k}{2}+1\right)}{\Gamma\left(\frac{1}{2}\right) \Gamma\left(\frac{k+1}{2}\right)} \sum_{r=k}^{\infty}(-1)^{r+k}\left(\begin{array}{l}
r \\
k
\end{array}\right) \frac{\left(\lambda t^{v}\right)^{r}}{\Gamma(v r+1)} \\
= & \sum_{k=1}^{\infty} \frac{k}{2} \frac{(1-w)^{k / 2} \Gamma\left(\frac{k}{2}\right)}{\Gamma\left(\frac{1}{2}\right) \Gamma\left(\frac{k+1}{2}\right)} \sum_{r=k}^{\infty}(-1)^{r+k}\left(\begin{array}{l}
r \\
k
\end{array}\right) \frac{\left(\lambda t^{v}\right)^{r}}{\Gamma(v r+1)}+\frac{1}{\pi} E_{v, 1}\left(-\lambda t^{v}\right)
\end{aligned}
$$




$$
\begin{aligned}
= & \sum_{k=1}^{\infty} \frac{k}{2} \frac{(1-w)^{k / 2} B\left(\frac{1}{2}, \frac{k}{2}\right)}{\pi} \sum_{r=k}^{\infty}(-1)^{r+k}\left(\begin{array}{l}
r \\
k
\end{array}\right) \frac{\left(\lambda t^{v}\right)^{r}}{\Gamma(v r+1)}+\frac{1}{\pi} E_{v, 1}\left(-\lambda t^{v}\right) \\
= & \frac{1}{2 \pi} \sum_{r=1}^{\infty} \frac{(-1)^{r}\left(\lambda t^{v}\right)^{r}}{\Gamma(v r+1)} \sum_{k=1}^{r} k(1-w)^{k / 2} B\left(\frac{1}{2}, \frac{k}{2}\right)(-1)^{k}\left(\begin{array}{l}
r \\
k
\end{array}\right)+\frac{1}{\pi} E_{v, 1}\left(-\lambda t^{v}\right) \\
= & \frac{1}{2 \pi} \sum_{r=1}^{\infty} \frac{(-1)^{r}\left(\lambda t^{v}\right)^{r}}{\Gamma(v r+1)} \int_{0}^{1} z^{-1 / 2}(1-z)^{-1} \sum_{k=1}^{r} k(-1)^{k}\left(\begin{array}{l}
r \\
k
\end{array}\right)(\sqrt{(1-z)(1-w)})^{k} d z \\
+ & \frac{1}{\pi} E_{v, 1}\left(-\lambda t^{v}\right) \\
= & \frac{1}{2 \pi} \sum_{r=1}^{\infty} \frac{(-1)^{r+1}\left(\lambda t^{v}\right)^{r}}{\Gamma(v r+1)} \int_{0}^{1} \frac{(1-w)^{1 / 2}}{z^{1 / 2}(1-z)^{1 / 2}} r(1-\sqrt{(1-z)(1-w)})^{r-1} d z \\
& +\frac{1}{\pi} E_{v, 1}\left(-\lambda t^{v}\right) \\
= & \frac{1}{2 \pi v} \sum_{r=1}^{\infty} \frac{(-1)^{r+1}\left(\lambda t^{v}\right)^{r}}{\Gamma(v r)} \int_{0}^{1} \frac{(1-w)^{1 / 2}}{z^{1 / 2}(1-z)^{1 / 2}}(1-\sqrt{(1-z)(1-w)})^{r-1} d z \\
& +\frac{1}{\pi} E_{v, 1}\left(-\lambda t^{v}\right) \\
& +\frac{1}{\pi} E_{v, 1}^{\infty}\left(-\lambda t^{v}\right) . \\
& \frac{1}{2 \pi v} \sum_{r=0}^{\infty} \frac{(-1)^{r}\left(\lambda t^{v}\right)^{r+1}}{\Gamma(v r+v)} \int_{0}^{1} \frac{(1-w)^{1 / 2}}{z^{1 / 2}(1-z)^{1 / 2}}(1-\sqrt{(1-z)(1-w)})^{r} d z \\
& \\
= &
\end{aligned}
$$

By inserting (3.25) into (3.24) we have that

$$
\begin{aligned}
& \operatorname{Pr}\left\{X\left(\mathscr{T}_{2 v}(t)\right) \in d x, Y\left(\mathscr{T}_{2 v}(t)\right) \in d y\right\} \\
= & \frac{d x d y}{\pi}\left\{\frac{\lambda t^{v}}{2 v} \int_{0}^{1} w^{-\frac{1}{2}} u_{2 v}\left(x, y, t ; c^{2} w\right) d w \int_{0}^{1}(z(1-z))^{-1 / 2}\right. \\
& \times \sum_{r=0}^{\infty} \frac{\left(-\lambda t^{v}\right)^{r}}{\Gamma(v r+v)}(1-\sqrt{(1-z)(1-w)})^{r} d z \\
& \left.+\int_{0}^{1}(w(1-w))^{-\frac{1}{2}} u_{2 v}\left(x, y, t ; c^{2} w\right) E_{v, 1}\left(-\lambda t^{v}\right) d w\right\} \\
= & \frac{d x d y}{\pi} \int_{0}^{1} w^{-\frac{1}{2}} u_{2 v}\left(x, y, t ; c^{2} w\right) d w\left\{\frac{E_{v, 1}\left(-\lambda t^{v}\right)}{(1-w)^{\frac{1}{2}}}\right. \\
& \left.+\frac{\lambda t^{v}}{2 v} \int_{0}^{1}(z(1-z))^{-1 / 2} E_{v, v}\left(-\lambda t^{v}(1-\sqrt{(1-z)(1-w)})\right) d z\right\}
\end{aligned}
$$

and result (3.23) easily follows. 
Remark 3.3 For $v=\frac{1}{2}$ formula (3.23) can be specialized, by considering (3.20), as follows:

$$
\begin{aligned}
& \operatorname{Pr}\{X(|B(t)|) \in d x, Y(|B(t)|) \in d y\} \\
= & \frac{d x d y}{\pi} \int_{0}^{1} \frac{e^{-\frac{x^{2}+y^{2}}{4 t c^{2} w}}}{4 \pi c^{2} w^{3 / 2} t} d w \\
& \times\left\{\frac{E_{\frac{1}{2}, 1}(-\lambda \sqrt{t})}{\sqrt{1-w}}+\lambda \sqrt{t} \int_{0}^{1} \frac{d z}{\sqrt{z(1-z)}} E_{\frac{1}{2}, \frac{1}{2}}(-\lambda \sqrt{t}(1-\sqrt{(1-w)(1-z)}))\right\} .
\end{aligned}
$$

An alternative form to (3.27) can be obtained by exploiting for $\operatorname{Pr}\{N(|B(t)|)=k\}$ the integral representation (2.23) instead of (2.10) and carrying out some steps based on (3.24)-(3.25):

$$
\begin{aligned}
& \operatorname{Pr}\{X(|B(t)|) \in d x, Y(|B(t)|) \in d y\} \\
& =d x d y\left\{\int _ { 0 } ^ { 1 } ( w ( 1 - w ) ) ^ { - \frac { 1 } { 2 } } \frac { e ^ { - \frac { x ^ { 2 } + y ^ { 2 } } { 4 c ^ { 2 } w } } } { 4 \pi c ^ { 2 } t w } d w \left[\frac{1}{\pi} E_{\frac{1}{2}, 1}(-\lambda \sqrt{t})+\right.\right. \\
& \left.\left.+\sum_{k=1}^{\infty} \frac{k B\left(\frac{1}{2}, \frac{k}{2}\right)}{2 \pi}(1-w)^{k / 2} \int_{0}^{\infty} e^{-\lambda v} \frac{(\lambda v)^{k}}{k !} \frac{e^{-\frac{v^{2}}{4 t}}}{\sqrt{\pi t}} d v\right]\right\} \\
& =d x d y\left\{\int _ { 0 } ^ { 1 } ( w ( 1 - w ) ) ^ { - \frac { 1 } { 2 } } \frac { e ^ { - \frac { x ^ { 2 } + y ^ { 2 } } { 4 c ^ { 2 } w } } } { 4 \pi c ^ { 2 } t w } d w \left[\frac{1}{\pi} E_{\frac{1}{2}, 1}(-\lambda \sqrt{t})+\right.\right. \\
& \left.\left.+\frac{1}{2 \pi} \int_{0}^{\infty} \frac{\lambda v e^{-\lambda v-\frac{v^{2}}{4 t}}}{\sqrt{\pi t}} d v \int_{0}^{1}(z(1-z))^{-\frac{1}{2}} e^{\lambda v \sqrt{1-w} \sqrt{1-z}} d z\right]\right\} .
\end{aligned}
$$

Remark 3.4 For $v=1$ formula (3.23) can be rewritten in a more suitable form as

$$
\begin{aligned}
& \operatorname{Pr}\left\{X\left(\mathscr{T}_{2}(t)\right) \in d x, Y\left(\mathscr{T}_{2}(t)\right) \in d y\right\} \\
= & \frac{d x d y e^{-\lambda t}}{\pi} \int_{0}^{1} \frac{u_{2}\left(x, y, t ; c^{2} w\right)}{w^{1 / 2}} d w\left[\frac{1}{\sqrt{1-w}}+\frac{\lambda t}{2} \int_{0}^{1} \frac{e^{\lambda t \sqrt{(1-w)(1-z)}} d z}{\sqrt{z(1-z)}}\right] \\
= & \frac{d x d y e^{-\lambda t}}{\pi} \int_{0}^{1} u_{2}\left(x, y, t ; c^{2} w\right) d w \\
& \times\left[\frac{1}{\sqrt{w} \sqrt{1-w}}+\frac{\lambda t}{2 \sqrt{w}} \sum_{m=0}^{\infty} \frac{(\lambda t)^{m}(1-w)^{\frac{m}{2}}}{m !} \int_{0}^{1} z^{-\frac{1}{2}}(1-z)^{\frac{m}{2}-\frac{1}{2}} d z\right],
\end{aligned}
$$

where $u_{2}\left(x, y, t ; c^{2} w\right)$ is given in (3.21). 
The expression in square brackets can be rewritten as follows

$$
\begin{aligned}
& \frac{1}{\sqrt{w} \sqrt{1-w}}+\frac{\lambda t}{2 \sqrt{w}} \sum_{m=0}^{\infty} \frac{(\lambda t \sqrt{1-w})^{m}}{m !} \frac{\Gamma\left(\frac{1}{2}\right) \Gamma\left(\frac{m}{2}+\frac{1}{2}\right)}{\Gamma\left(\frac{m}{2}+1\right)} \\
= & \frac{1}{\sqrt{w} \sqrt{1-w}}+\frac{\lambda t}{2 \sqrt{w}} \sum_{m=0}^{\infty} \frac{(\lambda t \sqrt{1-w})^{m}}{m} \frac{\pi 2^{1-m}}{\Gamma\left(\frac{m}{2}\right) \Gamma\left(\frac{m}{2}+1\right)} \\
= & \frac{1}{\sqrt{w} \sqrt{1-w}}+\frac{\pi \lambda t}{2 \sqrt{w}} \sum_{m=0}^{\infty}\left(\frac{\lambda t \sqrt{1-w}}{2}\right)^{m} \frac{1}{\Gamma^{2}\left(\frac{m}{2}+1\right)} \\
= & \frac{\pi \lambda t}{2 \sqrt{w}} \sum_{m=-1}^{\infty}\left(\frac{\lambda t \sqrt{1-w}}{2}\right)^{m} \frac{1}{\Gamma^{2}\left(\frac{m}{2}+1\right)},
\end{aligned}
$$

so that, by the change of variable $m+1=k$, (3.29) becomes

$$
\begin{aligned}
& \operatorname{Pr}\left\{X\left(\mathscr{T}_{2}(t)\right) \in d x, Y\left(\mathscr{T}_{2}(t)\right) \in d y\right\} \\
= & d x d y \frac{\lambda t e^{-\lambda t}}{2} \int_{0}^{1} \frac{u_{2}\left(x, y, t ; c^{2} w\right)}{\sqrt{w}} \sum_{k=0}^{\infty}\left(\frac{\lambda t}{2} \sqrt{1-w}\right)^{k-1} \frac{d w}{\Gamma^{2}\left(\frac{k}{2}+\frac{1}{2}\right)} .
\end{aligned}
$$

It can be easily checked that (3.30) integrates to one, by considering that $u_{2}\left(x, y, t ; c^{2} w\right)$ is a transition density and then $\iint_{\mathbb{R}^{2}} u_{2}\left(x, y, t ; c^{2} w\right) d x d y=1$.

By inserting formula (3.21) into (3.30) we get

$$
\begin{aligned}
& \operatorname{Pr}\left\{X\left(\mathscr{T}_{2}(t)\right) \in d x, Y\left(\mathscr{T}_{2}(t)\right) \in d y\right\} \\
= & d x d y \frac{\lambda t e^{-\lambda t}}{2} \int_{0}^{1} \frac{1}{2 \pi c w} \frac{\partial}{\partial t}\left[\frac{1_{C_{c t \sqrt{w}}}(x, y)}{\sqrt{c^{2} t^{2} w-\left(x^{2}+y^{2}\right)}}\right] \sum_{k=0}^{\infty}\left(\frac{\lambda t}{2} \sqrt{1-w}\right)^{k-1} \frac{d w}{\Gamma^{2}\left(\frac{k}{2}+\frac{1}{2}\right)} \\
= & {\left[\text { for } w t^{2}=z^{2}\right] } \\
= & d x d y \frac{\lambda t e^{-\lambda t}}{2} \int_{0}^{t} \frac{2 z t^{2}}{2 \pi c z^{2} t^{2}} \frac{z}{t} \frac{\partial}{\partial z}\left[\frac{1_{C_{c z}}(x, y)}{\sqrt{c^{2} z^{2}-\left(x^{2}+y^{2}\right)}}\right] \sum_{k=0}^{\infty}\left(\frac{\lambda}{2} \sqrt{t^{2}-z^{2}}\right)^{k-1} \frac{d z}{\Gamma^{2}\left(\frac{k}{2}+\frac{1}{2}\right)} \\
= & d x d y \frac{\lambda e^{-\lambda t}}{2 \pi c} \int_{0}^{t} \frac{\partial}{\partial z}\left[\frac{1_{C_{c z}}(x, y)}{\sqrt{c^{2} z^{2}-\left(x^{2}+y^{2}\right)}}\right] \sum_{k=0}^{\infty}\left(\frac{\lambda}{2} \sqrt{t^{2}-z^{2}}\right)^{k-1} \frac{d z}{\Gamma^{2}\left(\frac{k}{2}+\frac{1}{2}\right)}
\end{aligned}
$$

We can recognize in (3.31) the following expression

$$
\frac{\lambda e^{-\lambda t}}{2} \sum_{k=0}^{\infty}\left(\frac{\lambda \sqrt{t^{2}-u^{2}}}{2}\right)^{k-1} \frac{d u}{\Gamma^{2}\left(\frac{k}{2}+\frac{1}{2}\right)}=q(u ; t),
$$

which represents, for $|u|<t$, the distribution at time $t$ of the projection on the horizontal (or, equivalently, vertical) axis of the planar motion changing directions at Poisson times, performed at speed equal to 1 (see formula (1.3) in Orsingher and De Gregorio (2007), for $c=1$ ). This motion can also be regarded as a telegraph process changing velocity, randomly, at Poisson times (see, for details, Stadje and Zacks (2004)). 
Therefore we finally get

$$
\begin{aligned}
& \operatorname{Pr}\left\{X\left(\mathscr{T}_{2}(t)\right) \in d x, Y\left(\mathscr{T}_{2}(t)\right) \in d y\right\} \\
= & \frac{d x d y}{\pi c} \int_{0}^{t} \frac{\partial}{\partial z}\left[\frac{1_{C_{c z}}(x, y)}{\sqrt{c^{2} z^{2}-\left(x^{2}+y^{2}\right)}}\right] q(z ; t) d z \\
= & d x d y \int_{0}^{t} u_{2}\left(x, y, z ; c^{2}\right) q(z ; t) d z .
\end{aligned}
$$

The distribution (3.32) is concentrated in a circle of radius $c t$ and therefore has a different nature than all the other distributions (3.23), which are positive-valued on the whole plane. In effect (3.32) represents a cylindrical wave expanding on a circle with random radius, whose distribution is related to a telegraph process with random velocity.

\section{Alternative forms of fractional Poisson processes}

We present now two different versions of fractional Poisson processes obtained by following an approach alternative to that used in Section 2.

The first process we construct here, denoted by $\widehat{N}_{v}(t), t>0$, is obtained by replacing the factorial function in the Poisson distribution with the Gamma function and writing the probabilities as follows:

$$
\operatorname{Pr}\left\{\widehat{N}_{v}(t)=k\right\}=\frac{(\lambda t)^{k}}{\Gamma(v k+1)} \frac{1}{E_{v, 1}(\lambda t)}, \quad k \geq 0
$$

where $\lambda>0,0<v \leq 1$.

For $v=1$ the distribution (4.1) coincides with that of the homogeneous Poisson process. The process with one-dimensional distribution (4.1) can be viewed as a fractional version of the Poisson process because the Mittag-Leffler function emerges as the counterpart to the exponential function in the analysis of equations with fractional order derivatives. Nevertheless some basic properties of the Poisson process are lost when considering (4.1): in particular the independence of increments on non-overlapping intervals.

It is easily ascertained that

$$
\operatorname{Pr}\left\{T_{1}^{v}>t+s \mid T_{1}^{v}>s\right\}=\frac{E_{v, 1}(\lambda s)}{E_{v, 1}(\lambda(t+s))}
$$

where $T_{1}^{v}=\inf \left\{z: \widehat{N}_{v}(z)=1\right\}$ and thus (4.1) loses the lack of memory property of the Poisson distribution.

We note that the distribution (4.1) can be expressed in terms of the $k$-th factorial moments of the first-type fractional Poisson process $\mathscr{N}_{v}$, given in (2.9), as follows:

$$
\operatorname{Pr}\left\{\widehat{N}_{v}(t)=k\right\}=\frac{\mathbb{E}\left\{\mathscr{N}_{v}\left(t^{v}\right) \ldots\left[\mathscr{N}_{v}\left(t^{v}\right)-k+1\right]\right\}}{k ! E_{v, 1}(\lambda t)} .
$$


The probability generating function of (4.1) reads

$$
\begin{aligned}
G_{v}(u, t) & =\sum_{k=0}^{\infty} u^{k} \operatorname{Pr}\left\{\widehat{N}_{v}(t)=k\right\} \\
& =\frac{E_{v, 1}(u \lambda t)}{E_{v, 1}(\lambda t)}
\end{aligned}
$$

which can be compared with (2.6) of the first model.

We note that the function $G_{v}\left(u^{v}, t\right)$ is a solution to the fractional equation

$$
\frac{\partial^{v} G_{v}\left(u^{v}, t\right)}{\partial u^{v}}=\lambda t G_{v}\left(u^{v}, t\right), \quad 0<v<1,
$$

with the initial condition $G_{v}\left(u^{v}, 0\right)=1$.

Indeed we can check that

$$
\begin{aligned}
\frac{\partial^{v} G_{v}\left(u^{v}, t\right)}{\partial u^{v}} & =\frac{1}{E_{v, 1}(\lambda t)} \sum_{k=0}^{\infty} \frac{(\lambda t)^{k}}{\Gamma(v k+1)} \frac{1}{\Gamma(1-v)} \int_{0}^{u} \frac{d}{d v}\left[v^{v k}\right](u-v)^{-v} d v \\
& =\frac{1}{E_{v, 1}(\lambda t)} \sum_{k=1}^{\infty} \frac{(\lambda t)^{k} u^{v k-v}}{\Gamma(v(k-1)+1)}=\lambda t G_{v}\left(u^{v}, t\right) .
\end{aligned}
$$

The mean value of the distribution (4.1) is

$$
\mathbb{E} \widehat{N}_{v}(t)=\frac{\lambda t}{v} \frac{E_{v, v}(\lambda t)}{E_{v, 1}(\lambda t)}
$$

while the variance reads

$$
\begin{aligned}
& \operatorname{Var}_{v}(t) \\
= & \frac{\lambda t}{v^{2}} \frac{E_{v, v-1}(\lambda t)}{E_{v, 1}(\lambda t)}+\frac{\lambda t}{v^{2}} \frac{E_{v, v}(\lambda t)}{E_{v, 1}(\lambda t)}\left\{1-\lambda t \frac{E_{v, v}(\lambda t)}{E_{v, 1}(\lambda t)}\right\} \\
= & \frac{\lambda t}{v^{2}} \frac{E_{v, v-1}(\lambda t)}{E_{v, 1}(\lambda t)}+\frac{\mathbb{E} \widehat{N}_{v}(t)}{v}\left\{1-v \mathbb{E}_{v}(t)\right\} .
\end{aligned}
$$

We note that, for $v=1$, the expressions (4.6) and (4.7) coincide and thus

$$
\mathbb{E} \widehat{N}_{1}(t)=\operatorname{Var} \widehat{N}_{1}(t),
$$

as happens in the standard Poisson case.

We will consider now a sequence of a random number of non-negative i.i.d. random variables with distribution function $F(w)=\operatorname{Pr}\left(X_{i}<w\right), i \geq 1$, under the assumption that this random number is represented by $\widehat{N}_{v}(t)$. The distributions of the maximum and the minimum of this sequence can be written as follows

$$
\operatorname{Pr}\left\{\max \left(X_{1}, \ldots, X_{\widehat{N}_{v}(t)}\right)<w\right\}=\frac{E_{v, 1}(\lambda t F(w))}{E_{v, 1}(\lambda t)}
$$




$$
\operatorname{Pr}\left\{\min \left(X_{1}, \ldots, X_{\widehat{N}_{v}(t)}\right)>w\right\}=\frac{E_{v, 1}(\lambda t[1-F(w)])}{E_{v, 1}(\lambda t)} .
$$

We can compare result (4.8) with the analogous distribution obtained for the fractional Poisson process of the first type, $\mathscr{N}_{v}$, in (2.34): we note that they display similar structures and coincide for $v=1$.

Moreover we define a fractional counterpart of the compound Poisson process as follows

$$
Y_{\widehat{N}_{v}(t)}=\sum_{j=1}^{\widehat{N}_{v}(t)} X_{j}, \quad t>0
$$

and we obtain explicitly the characteristic function that satisfies the following relationship

$$
\begin{aligned}
\mathbb{E} e^{i \alpha Y_{\widehat{N}_{v}(t)}} & =\frac{E_{v, 1}\left(\lambda t \mathbb{E} e^{i \alpha X}\right)}{E_{v, 1}(\lambda t)} \\
& =G_{v}\left(\mathbb{E} e^{i \alpha X}, t\right) .
\end{aligned}
$$

The distribution of the waiting time of the first event $T_{1}^{v}$ is equal to

$$
\operatorname{Pr}\left\{T_{1}^{v}>t\right\}=\operatorname{Pr}\left\{\widehat{N}_{v}(t)=0\right\}=\frac{1}{E_{v, 1}(\lambda t)}
$$

For the waiting time of the $k$-th event $T_{k}^{v}$ we can write that

$$
\begin{aligned}
\operatorname{Pr}\left\{T_{k}^{v} \in d t\right\} & =\operatorname{Pr}\left\{\widehat{N}_{v}(t)=k-1, \widehat{N}_{v}[t, t+d t)=1\right\} \\
& =\operatorname{Pr}\left\{\widehat{N}_{v}(t)=k-1\right\}-\operatorname{Pr}\left\{\widehat{N}_{v}(t)=k-1, \widehat{N}_{v}[t, t+d t) \neq 1\right\}
\end{aligned}
$$

where we assume that $\left\{\widehat{N}_{v}[t, t+d t) \neq 1\right\}=\left\{\widehat{N}_{v}[t, t+d t)=0\right\}$, up to some infinitesimal function of $d t$.

We construct now the third version of the fractional Poisson process (which we will denote by $\bar{N}_{v}(t), t>0$ ), by assuming that its interarrival times $U_{j}$ coincide in distribution with the waiting time of the first event of the first model, i.e. $T_{1}^{v}=\inf \left\{t: \mathscr{N}_{v}(t)=1\right\}$. Therefore we deduce the distribution of the r.v.'s representing the interarrival times, $U_{j}, j \geq 1$, from (2.11), as follows

$$
\operatorname{Pr}\left\{U_{j}>t\right\}=\operatorname{Pr}\left\{T_{1}^{v}>t\right\}=\operatorname{Pr}\left\{\mathscr{N}_{v}(t)=0\right\}=E_{v, 1}\left(-\lambda t^{v}\right)
$$

and then (in view of (5.2) in Appendix)

$$
\operatorname{Pr}\left\{U_{j} \in d t\right\}=\lambda t^{v-1} E_{v, v}\left(-\lambda t^{v}\right) d t,
$$

for any $j \geq 1, \lambda>0, t>0$ and $0<v \leq 1$.

By taking the Laplace transform of (4.14) we get

$$
\int_{0}^{+\infty} e^{-\mu t} \operatorname{Pr}\left\{U_{j} \in d t\right\}=\frac{\lambda}{\lambda+\mu^{v}}, \quad \mu>0 .
$$


Therefore the moment-generating function of the waiting time of the $m$-th event $T_{m}^{v}=U_{1}+U_{2}+$ $\ldots+U_{m}$ can be rewritten as

$$
\begin{aligned}
\mathbb{E} e^{-\mu T_{m}^{v}} & =\left(\frac{\lambda}{\lambda+\mu^{v}}\right)^{m} \\
& =\int_{0}^{+\infty} e^{-\mu^{v} t} \lambda^{m} \frac{t^{m-1} e^{-\lambda t}}{\Gamma(m)} d t \\
& =\int_{0}^{+\infty} e^{-\mu^{v} t} g(t ; \lambda, m) d t
\end{aligned}
$$

where $g(t ; \lambda, m)$ is a Gamma density of parameters $\lambda, m$.

We can obtain the distribution of the new process $\bar{N}_{v}(t), t>0$, from that of the interarrival times $U_{j}$, as follows:

$$
\begin{aligned}
& \operatorname{Pr}\left\{\bar{N}_{v}(t)=m\right\} \\
= & \operatorname{Pr}\left\{U_{1}+\ldots+U_{m}<t, U_{1}+\ldots+U_{m+1}>t\right\} \\
= & \operatorname{Pr}\left\{U_{1}+\ldots+U_{m}<t\right\}-\operatorname{Pr}\left\{U_{1}+\ldots+U_{m+1}<t\right\} \\
= & \operatorname{Pr}\left\{T_{m}^{v}<t\right\}-\operatorname{Pr}\left\{T_{m+1}^{v}<t\right\} .
\end{aligned}
$$

From the analysis presented above we see that the r.v.'s representing the increments between two successive occurrences, i.e. $\overline{\mathscr{N}}_{v}\left(T_{m}^{v}\right)-\overline{\mathscr{N}}_{v}\left(T_{m-1}^{v}\right), m \geq 1$, are independent. However, since, for arbitrary time instants $t_{j-1}, t_{j}$, the r.v.'s $\overline{\mathscr{N}}_{v}\left(t_{j}\right)-\overline{\mathscr{N}}_{v}\left(t_{j-1}\right)$ are not independent, the process is not endowed with independent increments.

Remark 4.1 We note that, from (4.17),

$$
\begin{aligned}
& \int_{0}^{\infty} e^{-\mu t} \operatorname{Pr}\left\{\bar{N}_{v}(t)=k\right\} \\
= & \int_{0}^{\infty} e^{-\mu t}\left[\int_{0}^{t} \operatorname{Pr}\left\{U_{1}+\ldots+U_{k} \in d s\right\}-\int_{0}^{t} \operatorname{Pr}\left\{U_{1}+\ldots+U_{k+1} \in d s\right\}\right] d t \\
= & \frac{1}{\mu} \int_{0}^{\infty} e^{-\mu s}\left[\operatorname{Pr}\left\{U_{1}+\ldots+U_{k} \in d s\right\}-\operatorname{Pr}\left\{U_{1}+\ldots+U_{k+1} \in d s\right\}\right] \\
= & \frac{1}{\mu}\left[\left(\frac{\lambda}{\lambda+\mu^{v}}\right)^{k}-\left(\frac{\lambda}{\lambda+\mu^{v}}\right)^{k+1}\right]=\frac{\mu^{v-1} \lambda^{k}}{\left(\lambda+\mu^{v}\right)^{k+1}} .
\end{aligned}
$$

This coincides with the result of Remark 2.4 and thus the one-dimensional distributions of the first and third models coincide.

Another representation of the distribution of the fractional Poisson process is given in the next theorem.

Theorem 4.1 Let $f_{v}(\cdot ; t)$ be the density of the stable r.v. $S_{v}(\mu, \beta, \sigma)$, where $\mu=0, \beta=1$ and $\sigma=(t \cos \pi v / 2)^{1 / v}$, then the distribution of the process $\bar{N}_{v}(t), t>0$ defined by (4.17) and (4.13) is 
given by

$$
\begin{aligned}
& \operatorname{Pr}\left\{\overline{\mathscr{N}}_{v}(t)=m\right\} \\
= & \int_{0}^{t} d z \int_{0}^{\infty} f_{v}(z ; u)\left[\frac{e^{-\lambda u} \lambda^{m} u^{m-1}}{(m-1) !}-\frac{e^{-\lambda u} \lambda^{m+1} u^{m}}{m !}\right] d u \\
= & \lambda \int_{0}^{t} d z \int_{0}^{\infty} f_{v}(z ; u)[\operatorname{Pr}\{N(u)=m-1\}-\operatorname{Pr}\{N(u)=m\}] d u,
\end{aligned}
$$

for $m \geq 1$ and

$$
\operatorname{Pr}\left\{\overline{\mathscr{N}}_{v}(t)=0\right\}=E_{v, 1}\left(-\lambda t^{v}\right) .
$$

Proof In order to write down explicitly the distribution (4.17) we recall that the factor $e^{-\mu^{v} t}$ appearing in (4.16) is the Laplace transform of the stable law $f_{v}(\cdot ; t)$ (see (2.19)), therefore (4.16) can be rewritten as

$$
\mathbb{E} e^{-\mu T_{m}^{v}}=\int_{0}^{+\infty}\left(\int_{0}^{\infty} e^{-\mu z} f_{v}(z ; t) d z\right) g(t ; \lambda, m) d t .
$$

From (4.20) we can infer that, for $m \geq 1$,

$$
\operatorname{Pr}\left\{T_{m}^{v} \in d z\right\}=d z \int_{0}^{\infty} f_{v}(z ; t) g(t ; \lambda, m) d t, \quad z \geq 0 .
$$

and the first expression in (4.18) easily follows. Formula (4.19) is derived by considering (4.13).

Remark 4.2 By considering the equation governing the standard Poisson process, we can rewrite (4.18) as

$$
\begin{aligned}
& \operatorname{Pr}\left\{\bar{N}_{v}(t)=m\right\} \\
= & \int_{0}^{t} d z \int_{0}^{\infty} f_{v}(z ; u) \frac{d}{d u} \operatorname{Pr}\{N(u)=m\} d u \\
= & -\int_{0}^{t} d z \int_{0}^{\infty} \frac{\partial}{\partial u} f_{v}(z ; u) \operatorname{Pr}\{N(u)=m\} d u .
\end{aligned}
$$

In the standard case $v=1$ it is easy to check that (4.21) reduces to the Gamma distribution representing the waiting time of the $m$-th event, since $f_{1}(z ; u)=\delta(z-u)$ :

$$
\begin{aligned}
& \operatorname{Pr}\left\{T_{m} \in d z\right\}=d z \int_{0}^{\infty} \delta(z-u) g(u ; \lambda, m) d u \\
= & g(z ; \lambda, m) d z,
\end{aligned}
$$

while (4.18) and (4.19) coincide with the standard Poisson distribution:

$$
\begin{aligned}
& \operatorname{Pr}\left\{\bar{N}_{1}(t)=m\right\} \\
= & \lambda \int_{0}^{t} d z \int_{0}^{\infty} \delta(z-u)[\operatorname{Pr}\{N(u)=m-1\}-\operatorname{Pr}\{N(u)=m\}] d u \\
= & \lambda \int_{0}^{t}[\operatorname{Pr}\{N(z)=m-1\}-\operatorname{Pr}\{N(z)=m\}] d z \\
= & \operatorname{Pr}\{N(t)=m\}
\end{aligned}
$$


for $m \geq 1$ and

$$
\operatorname{Pr}\left\{\overline{\mathscr{N}}_{1}(t)=0\right\}=e^{-\lambda t}
$$

Remark 4.3 For $v=\frac{1}{2}$, the density $f_{\frac{1}{2}}(z ; t)$ has the following explicit form

$$
f_{\frac{1}{2}}(z ; t)=\frac{t}{\sqrt{2}} \frac{e^{-\frac{t^{2}}{4 z}}}{\sqrt{2 \pi z^{3}}}, \quad z, t>0
$$

which coincides with the limiting case, for $\mu \rightarrow \infty$, of the Inverse Gaussian distribution, defined as

$$
f(z ; t)=\sqrt{\frac{\lambda}{2 \pi z^{3}}} e^{-\frac{\lambda(z-\mu)^{2}}{4 \mu^{2} z}}, \quad \lambda, z>0,
$$

for $\lambda=t^{2} / 2$.

Therefore, for $v=\frac{1}{2}$ and $m \geq 1$, (4.21) reduces to

$$
\operatorname{Pr}\left\{T_{m}^{1 / 2} \in d z\right\}=\frac{d z}{(m-1) !} \int_{0}^{\infty} \frac{\lambda^{m} t^{m}}{\sqrt{2}} \frac{e^{-\frac{t^{2}}{4 z}-\lambda t}}{\sqrt{2 \pi z^{3}}} d t, \quad z \geq 0
$$

Analogously the distribution of the process given in (4.18)-(4.19) becomes, for $v=\frac{1}{2}$,

$$
\begin{aligned}
& \operatorname{Pr}\left\{\bar{N}_{1 / 2}(t)=m\right\} \\
= & \frac{\lambda}{2 \sqrt{\pi}} \int_{0}^{t} d z \int_{0}^{\infty} e^{-\lambda u} \frac{u e^{-\frac{u^{2}}{4 z}}}{\sqrt{z^{3}}}\left[\frac{(\lambda u)^{m-1}}{(m-1) !}-\frac{(\lambda u)^{m}}{m !}\right] d u,
\end{aligned}
$$

for $m \geq 1$ and

$$
\operatorname{Pr}\left\{\overline{\mathscr{N}}_{1 / 2}(t)=0\right\}=E_{\frac{1}{2}, 1}(-\lambda \sqrt{t}) .
$$

Formula (4.24) suggests a first interpretation of the distribution, in terms of a Rayleigh r.v. $R_{z}$, i.e.

$$
\operatorname{Pr}\left\{R_{z} \in d u\right\}=\frac{u e^{-\frac{u^{2}}{4 z}} d u}{2 z}, \quad z, u>0
$$

as

$$
\begin{aligned}
& \operatorname{Pr}\left\{\bar{N}_{1 / 2}(t)=m\right\} \\
= & \frac{\lambda}{\sqrt{\pi}} \int_{0}^{t} \frac{d z}{\sqrt{z}}\left[\operatorname{Pr}\left\{N\left(R_{z}\right)=m-1\right\}-\operatorname{Pr}\left\{N\left(R_{z}\right)=m\right\}\right] .
\end{aligned}
$$


We can obtain a further alternative expression of the distribution $\operatorname{Pr}\left\{\overline{\mathscr{N}}_{1 / 2}(t)=m\right\}, m \geq 1$, in terms of the first model, by means of an integration by parts in (4.24):

$$
\begin{aligned}
& \operatorname{Pr}\left\{\bar{N}_{1 / 2}(t)=m\right\} \\
= & \frac{\lambda^{m}}{(m-1) ! \sqrt{\pi}} \int_{0}^{t} \frac{d z}{\sqrt{z}} \int_{0}^{\infty} e^{-\lambda u} \frac{u e^{-\frac{u^{2}}{4 z}}}{2 z}\left[u^{m-1}-\frac{\lambda u^{m}}{m}\right] d u \\
= & \frac{\lambda^{m}}{(m-1) ! \sqrt{\pi}} \int_{0}^{t} \frac{d z}{\sqrt{z}} \int_{0}^{\infty} e^{-\lambda u} e^{-\frac{u^{2}}{4 z}}\left[(m-1) u^{m-2}-2 \lambda u^{m-1}+\frac{\lambda^{2} u^{m}}{m}\right] d u \\
= & \lambda^{2} \int_{0}^{t} d z \int_{0}^{\infty} \frac{e^{-\frac{u^{2}}{4 z}}}{\sqrt{\pi z}} e^{-\lambda u}\left[\frac{(\lambda u)^{m-2}}{(m-2) !}-\frac{2(\lambda u)^{m-1}}{(m-1) !}+\frac{(\lambda u)^{m}}{m !}\right] d u \\
= & \lambda^{2} \int_{0}^{t} d z \int_{0}^{\infty} \frac{e^{-\frac{u^{2}}{4 z}}}{\sqrt{\pi z}}[\operatorname{Pr}\{N(u)=m-2\}-2 \operatorname{Pr}\{N(u)=m-1\}+\operatorname{Pr}\{N(u)=m\}] d u \\
= & \lambda^{2} \int_{0}^{t}\left[\operatorname{Pr}\left\{\mathscr{N}_{1 / 2}(z)=m-2\right\}-2 \operatorname{Pr}\left\{\mathscr{N}_{1 / 2}(z)=m-1\right\}+\operatorname{Pr}\left\{\mathscr{N}_{1 / 2}(z)=m\right\}\right] d z,
\end{aligned}
$$

where, in the last step, we have used result (2.23).

If we now take the first derivative of (4.26) we obtain

$$
\frac{d}{d t} \operatorname{Pr}\left\{\bar{N}_{1 / 2}(t)=m\right\}=\lambda\left[\frac{d^{1 / 2}}{d t^{1 / 2}} \operatorname{Pr}\left\{\mathscr{N}_{1 / 2}(t)=m-1\right\}-\frac{d^{1 / 2}}{d t^{1 / 2}} \operatorname{Pr}\left\{\mathscr{N}_{1 / 2}(t)=m\right\}\right]
$$

by considering that $\operatorname{Pr}\left\{\mathscr{N}_{1 / 2}(t)=m\right\}$ solves equation (2.1), for $v=\frac{1}{2}$.

\section{Appendix}

We note that the following identity for the Mittag-Leffler function hold:

$$
\frac{1}{x} E_{-v, 1-v}\left(\frac{1}{x}\right)=-E_{v, 1}(x),
$$

for $0<v<1$ and $x \neq 0$. In order to prove (5.1) we use the contour integral representation of the inverse of the Gamma function, so that we can write

$$
\begin{aligned}
& \frac{1}{x} \sum_{k=0}^{\infty} \frac{\left(\frac{1}{x}\right)^{k}}{\Gamma(-v k+1-v)} \\
= & \frac{1}{x} \frac{1}{2 \pi i} \sum_{k=0}^{\infty}\left(\frac{1}{x}\right)^{k} \int_{H a} e^{t} t^{-1+v k+v} d t \\
= & \frac{1}{x} \frac{1}{2 \pi i} \int_{H a} e^{t} t^{-1+v} \frac{1}{1-\frac{t^{v}}{x}} d t \\
= & \frac{1}{2 \pi i} \int_{H a} \frac{e^{t} t^{-1+v}}{x-t^{v}} d t=-E_{v, 1}(x) .
\end{aligned}
$$


For the derivatives of the Mittag-Leffler function the following results hold

$$
\begin{aligned}
\frac{d}{d x} E_{v, 1}(x) & =\frac{1}{v} E_{v, v}(x) \\
\frac{d}{d x} E_{v, v}(x) & =\frac{1}{v} E_{v, 2 v-1}(x)+\frac{1-v}{v} E_{v, 2 v}(x),
\end{aligned}
$$

as can be checked by simple manipulations.

We can also show that

$$
\begin{aligned}
& E_{\mu,-v}(x) \\
= & x^{m} E_{\mu, \mu m-v}(x)+\frac{1}{\Gamma(-v)}+\frac{x}{\Gamma(\mu-v)}+\ldots+\frac{x^{m-1}}{\Gamma(\mu(m-1)-v)},
\end{aligned}
$$

for $\mu, v>0$ and for any integer $m>1$. The relationship (5.3) can be derived as follows

$$
\begin{aligned}
& \sum_{r=-m}^{E_{\mu,-v}(x)} \frac{x^{r+m}}{\Gamma(\mu(r+m)-v)} \\
= & \sum_{r=0}^{\infty} \frac{x^{r+m}}{\Gamma(\mu(r+m)-v)}+\frac{1}{\Gamma(-v)}+\frac{x}{\Gamma(\mu-v)}+\ldots+\frac{x^{m-1}}{\Gamma(\mu(m-1)-v)} .
\end{aligned}
$$

\section{Acknowledgements}

Thanks are due to the referee for the accurate reading of the first draft of this manuscript, which led to a substantial improvement of the paper. We recently learned about the paper by Mainardi, Gorenflo, Scalas (2004), Vietnam Journal of Mathematics, p.53-64, with some intersections with our work.

\section{References}

[1] Jumarie G. (2001), Fractional master equation: non-standard analysis and Liouville-Riemann derivative, Chaos, Solitons and Fractals, 12, 2577-2587, MR1851079 (2003i:82069).

[2] Kolesnik A., Orsingher, E. (2005), A planar random motion with an infinite number of directions controlled by the damped wave equation, Journ. Appl. Prob., 42, (4), 1168-1182, MR2203830 (2007a:60046).

[3] Laskin, N. (2003), Fractional Poisson process, Communications in Nonlinear Science and Numerical Simulation, 8, 201-213, MR2007003 (2004j:60101).

[4] Orsingher, E. (1985), Vibrations with random initial conditions, Bollettino dell'Unione Matematica Italiana, 6, 4-B, 541-556, MR0805427 (87f:60093). 
[5] Orsingher, E., Beghin, L. (2004), Time-fractional equations and telegraph processes with Brownian time, Probability Theory and Related Fields, 128, 141-160, MR2027298 (2005a:60056).

[6] Orsingher, E., Beghin, L. (2009), Fractional diffusion equations and processes with randomly-varying time, Annals of Probability, 37 (1), 206-249, MR2489164.

[7] Orsingher, E., De Gregorio A. (2007), Random flights in higher spaces, Journ. Theor. Prob., 20, n.4, 769-806, MR2359055 (2008h:60041).

[8] Podlubny, I. (1999), Fractional Differential Equations, Academic Press, San Diego, MR1658022 (99m:26009).

[9] Repin O.N., Saichev, A.I. (2000), Fractional Poisson law, Radiophysics and Quantum Electronics, 43 (9), 738-741, MR1910034.

[10] Samko, S.G., Kilbas, A.A., Marichev, O.I. (1993), Fractional Integrals and Derivatives: Theory and Applications, Gordon and Breach Science Publishers, MR1347689 (96d:26012).

[11] Smirnov V.I. (1981), Corso di Matematica Superiore, vol.II, Ed. Riuniti.

[12] Stadje, W., Zacks, S. (2004), Telegraph processes with random velocities, Journ. Appl. Prob., 3, 665-678, MR2074815 (2005g:60116).

[13] Wang, X.T., Wen, Z.X., Zhang, S.Y. (2006), Fractional Poisson process (II), Chaos, Solitons and Fractals, 28, 143-147, MR2174587.

[14] Wang, X.T., Zhang, S.Y., Fan S. (2007), Non-homogeneous fractional Poisson processes, Chaos, Solitons and Fractals, 31, 236-241, MR2263284. 